\section{(6) OPEN ACCESS}

- Additional material is published online only. To view please visit the journal online (http://dx.doi.org/10.1136/ gutjnl-2016-311393).

For numbered affiliations see end of article.

\section{Correspondence to}

Professor Julia Mayerle, Department of Medicine A, University Medicine, ErnstMoritz-Arndt-University, Greifswald, FerdinandSauerbruchstrasse, Greifswald 17475, Germany; mayerle@ uni-greifswald.de

Received 3 January 2016 Revised 1 April 2016 Accepted 18 April 2016 Published Online First 12 May 2016

\section{SLinked}

- http://dx.doi.org/10.1136/ gutjnl-2016-312092

\title{
Tumour-specific delivery of siRNA-coupled superparamagnetic iron oxide nanoparticles, targeted against PLK1, stops progression of pancreatic cancer
}

\author{
Ujjwal M Mahajan, ${ }^{1}$ Steffen Teller, ${ }^{1}$ Matthias Sendler, ${ }^{1}$ Raghavendra Palankar, ${ }^{2}$ \\ Cindy van den Brandt, ${ }_{1}^{1}$ Theresa Schwaiger, ${ }^{1}$ Jens-Peter Kühn, ${ }^{3}$ Silvia Ribback, ${ }^{4}$ \\ Gunnar Glöckl, ${ }^{5}$ Matthias Evert, ${ }^{4}$ Werner Weitschies, ${ }^{5}$ Norbert Hosten, ${ }^{3}$ \\ Frank Dombrowski, ${ }^{5}$ Mihaela Delcea, ${ }^{2}$ Frank-Ulrich Weiss, ${ }^{1}$ Markus M Lerch, ${ }^{1}$ \\ Julia Mayerle ${ }^{1}$
}

\begin{abstract}
Objective Pancreatic ductal adenocarcinoma (PDAC) is one of the most aggressive malignancies and is projected to be the second leading cause of cancerrelated death by 2030 . Despite extensive knowledge and insights into biological properties and genetic aberrations of PDAC, therapeutic options remain temporary and ineffective. One plausible explanation for the futile response to therapy is an insufficient and non-specific delivery of anticancer drugs to the tumour site.

Design Superparamagnetic iron oxide nanoparticles (SPIONs) coupled with siRNA directed against the cell cycle-specific serine-threonine-kinase, Polo-like kinase-1 (siPLK1-StAv-SPIONs), could serve a dual purpose for delivery of siPLK1 to the tumour and for non-invasive assessment of efficiency of delivery in vivo by imaging the tumour response. siPLK1-StAv-SPIONs were designed and synthesised as theranostics to function via a membrane translocation peptide with added advantage of driving endosomal escape for mediating transportation to the cytoplasm (myristoylated polyarginine peptides) as well as a tumour-selective peptide (EPPT1) to increase intracellular delivery and tumour specificity, respectively.
\end{abstract}

Results A syngeneic orthotopic as well as an endogenous cancer model was treated biweekly with siPLK1-StAv-SPIONs and tumour growth was monitored by small animal MRI. In vitro and in vivo experiments using a syngeneic orthotopic PDAC model as well as the endogenous LSL-Kras ${ }^{\mathrm{G} 12 \mathrm{D}}$, LSL-Trp53 ${ }^{\mathrm{R} 172 \mathrm{H}}$, Pdx-1-Cre model revealed significant accumulation of siPLK1-StAvSPIONs in PDAC, resulting in efficient PLK1 silencing. Tumour-specific silencing of PLK1 halted tumour growth, marked by a decrease in tumour cell proliferation and an increase in apoptosis.

Conclusions Our data suggest siPLK1-StAv-SPIONs with dual specificity residues for tumour targeting and membrane translocation to represent an exciting opportunity for targeted therapy in patients with PDAC.

\section{INTRODUCTION}

Pancreatic ductal adenocarcinoma (PDAC) is one of the most aggressive malignancy, burdened with a

\section{Significance of this study}

What is already known on this subject?

- Pancreatic ductal adenocarcinoma is one of the most aggressive malignancies with transitory therapeutic effectiveness to chemotherapeutics drugs, owing to insufficient delivery of these drugs to the tumour site.

- Cancer cells overexpress underglycosylated MUC1 (uMUC1). These molecules extend 100$200 \mathrm{~nm}$ above the surface, making uMUC1 an accessible target for targeted delivery of therapeutic probes.

- Owing to its specificity, selectivity and better tolerability with minimal side effects, siRNAs provide the basis for development of selective anticancer therapy compared with the relative promiscuity of small-molecule inhibitors.

- Superparamagnetic iron oxide nanoparticles (SPIONs) are of potential use for targeted drug delivery and biomedical imaging applications.

\section{What are the new findings?}

- Both the uMUC1-specific ligand, EPPT1 and the myristoylated polyarginine peptide ligand are essential for tumour-specific targeted delivery of siPLK1 through the aid of SiPLK1-StAv-SPIONs.

- siPLK1-StAv-SPIONs provide a multifunctional theranostic platform for tumour-specific targeted delivery and simultaneous MRI that allow monitoring of tumour response and siPLK1 treatment efficacy.

- siPLK1-StAv-SPIONs stop tumour progression by silencing of siPLK1 in two clinically relevant pancreatic cancer models. 
Significance of this study

How might it impact on clinical practice in the foreseeable future?

- siPLK1-StAv-SPIONs treatment represents an important step towards the application of siRNAs as cancer therapeutic agents by providing an imaging strategy and overcoming systemic dose limitations of small-molecule inhibitors directed against PLK1.

- Tumour-specific targeting by means of siPLK1-StAv-SPIONs shows promising potential as companion diagnostic.

optimising drug delivery to the tumour. Given the importance of the regulation of the cell division cycle, alteration of the cell cycle is a hallmark feature of human malignancies. ${ }^{2}$ Many of the genes encoding regulators of the cell cycle are mutated in pancreatic cancers. ${ }^{3}$ Considering the role of Polo-like kinases (PLKs) in the centrosome cycle, dysregulated expression of PLKs is associated with oncogenesis. ${ }^{4}$ Overexpression of PLK1 was detected in both pancreatic cancer cell lines and in resection specimens of PDAC. ${ }^{5}$ Furthermore, PLK1 is differentially expressed in normal and cancer tissue, making it an ideal target for cancer-specific silencing. ${ }^{6}$

Several PLK1-specific inhibitors have been tested in clinical trials, but owing to their non-specificity and very high toxicity, their clinical use was shown to be limited. ${ }^{7}$ The use of siRNAs to target PLK1 would allow specific intervention at the level of a single molecule and may provide an advantage overcoming the non-specificity of small-molecule inhibitors. Specific targeting of PLK1 through the aid of siRNAs holds much promise for the treatment of a wide range of human cancers. ${ }^{8-10}$ However, major hurdles of in vivo delivery of siRNAs are the inadequate delivery to the specific site of interest, stability of siRNAs in serum and monitoring of siRNA delivery.

The objective of the present study in parallel to the above outlined concepts is to evaluate the potential of siRNA conjugated dual-purpose superparamagnetic iron oxide nanoparticles (SPIONs) for the therapeutic intervention of PDAC that function as theranostics. Although the feasibility of using SPIONs for cancer detection and drug delivery has been demonstrated, ${ }^{11}$ a major obstacle limiting their clinical application is that nontargeted nanoparticles are unable to reach sufficient concentrations at the tumour site to either produce a strong signal for tumour imaging or to carry optimal amounts of therapeutic agents into tumour cells.

We developed multifunctional theranostic probes comprising of dextran-coated SPIONs, which can be detected by MRI and which are functionalised through (i) the non-immunogenic ligand EPPT1 specifically targeting the tumour antigen underglycosylated MUC1 (uMUC1), a hallmark of early pancreatic tumorigenesis and abundantly and ubiquitously expressed in PDAC; (ii) the ligand myristoylated polyarginine peptides (MPAPs) to enhance cellular uptake by electrostatic interaction and mediating the endosomal escape, thus releasing siPLK1 into the cytoplasm; and (iii) siPLK1, for selective silencing of siPLK1 to halt cell cycle progression selectively in the tumour. Administrations of these selective PLK1 targeting complex, siPLK1-StAv-SPIONs, in tumour-bearing mice allow monitoring of delivery of the agent to the tumour by MRI and result in efficient silencing of the target gene, PLK1. This approach can significantly advance the therapeutic potential of siRNAs by providing a way to effectively shuttle siRNAs to target sites and to non-invasively access therapeutic efficacy of the siRNAs by monitoring tumour growth.

\section{MATERIALS AND METHODS}

Detailed methodology is described in the online supplementary methods. Briefly, the synthesis of siPLK1-StAv-SPIONs was performed according to the method adopted and modified from US patent $5262176 .{ }^{12}$ Details of synthesis, physical characterisation and in vitro experiments for iron quantification, cell cycle analysis by propidium iodide staining and $\mathrm{BrdU}+$ proliferation are provided in online supplementary methods. All animals' studies were complied with the National Institute of Health $(\mathrm{NIH})$ guidelines on handling of experimental animals. C57BL/6N mice were purchased from Charles-River, Sulzfeld, Germany, and housed under standard conditions. 6606PDA cell line was implanted in animals by orthotopic injection in the head of the pancreas as described previously. ${ }^{13}$ Fourteen days after tumour implantation, animals were randomised to three groups to receive $3 \mathrm{qD}^{*} 4$ weeks intravenous injections of siPLK1 $(100 \mathrm{nM} / \mathrm{kg})$, siControl-StAv-SPIONs $(5 \mathrm{mg} / \mathrm{kg}$ of iron) or siPLK1-StAv-SPIONs $(5 \mathrm{mg} / \mathrm{kg}$ of iron), respectively. At the end of treatment regimen, animals were euthanased and tissue harvested for further investigation. The LSL-Kras ${ }^{\mathrm{G} 12 \mathrm{D}}$,LSL-Trp53 ${ }^{\mathrm{R} 172 \mathrm{H}}$,Pdx-1-Cre (KPC) strains of mice have been generated as previously reported. ${ }^{14}$ Also, $16-$ 20 -week-old mice were randomised into three groups. Animals were treated in a randomised double-blind study for 2 weeks to receive $3 \mathrm{qD}^{*} 2$ weeks intravenous injection of StAv-SPIONs $(5 \mathrm{mg} / \mathrm{kg}$ of iron), siControl-StAv-SPIONs $(5 \mathrm{mg} / \mathrm{kg}$ of iron) or siPLK1-StAv-SPIONs $(5 \mathrm{mg} / \mathrm{kg}$ of iron), respectively. Tumour growth was monitored by ultrasound imaging as previously described. ${ }^{15}$ For fluorescent live cell imaging siPLK1-StAv-SPIONs were further conjugated with biotinylated Cy5 (Nanoc, New York, USA). Cells were treated with $50 \mu \mathrm{M}$ of siPLK1-StAv-SPIONs and live cell confocal laser scanning microscopy was carried out for $30 \mathrm{~min}$ at $37^{\circ} \mathrm{C}$ under controlled atmosphere on Olympus FLUOVIEW FV1000 microscope. To determine uptake mechanism, cells were preincubated for $30 \mathrm{~min}$ with anti-MUC1 antibody (Cell Signaling, Danvers, USA) and 30 min with Dynasore respectively prior to $30 \mathrm{~min}$ treatment of Cy5-coupled siPLK1-StAv-SPIONs. Image processing and analysis were performed with CellSens (Olympus, Tokyo, Japan) software. For transmission electron microscopy (TEM), cells were grown on polycarbonate mesh and at 0,5 and 30 min treatment with siPLK1-StAv-SPIONs and fixed as described previously. ${ }^{16}$ Ultrathin sections were stained with uranyl acetate and lead citrate and examined with a Libra 120 electron microscope from Carl Zeiss (Jena, Germany).

In vivo $7 \mathrm{~T}$ small animal MRI was performed as described previously. ${ }^{13}$ Quantitative determination of iron overload in tumour was calculated using $\mathrm{R}_{2}{ }^{*}$ fat-corrected single-peak echos collected during MRI using Osirix Diacom viewer software. Immunoblotting for anti-mouse PLK1 (Thermo Scientific, Massachusetts, USA), anti-mouse proliferating cell nuclear antigen (PCNA) (Invitrogen, Carlsbad, USA) and anti-mouse glyceraldehyde 3-phosphate dehydrogenase (GAPDH) (Meridian Life Sciences, Tennessee, USA) were performed as described previously. ${ }^{17}$ Immunostaining for anti-mouse Ki67 (Bethyl Laboratories, Texas, USA) and anti-mouse PLK1 (Thermo Scientific) were performed in accordance with the previously described procedure. ${ }^{17}$ For survival analysis, tumour-bearing mice were enrolled 14 days after syngeneic orthotopic tumour implantation. Animals were randomised into three groups to receive siControl-StAv-SPIONs 
(mismatch control-RNAi), gemcitabine or siPLK1-StAv-SPIONs, respectively. BI6727 (intraperitoneally, $25 \mathrm{mg} / \mathrm{kg} /$ week). siControl-StAv-SPIONs and siPLK1-StAv-SPIONs (intravenously, $5 \mathrm{mg} / \mathrm{kg}$ of iron) were administered twice weekly for maximum 12 weeks after initiation of the treatment. Objective response was monitored daily by visual examination of the animals and end point criteria were defined as general morbidity, lethargy or lack of social interaction. All the data are expressed as mean \pm SD unless otherwise stated and analysed using GraphPad (Prism 5.0, California, USA). $\mathrm{p}<0.05$ was considered statistically significant.

\section{RESULTS \\ Characterisation of siPLK1-StAv-SPIONs and their influence on cancer cell growth}

As part of a systematic effort to minimise the limitations of classical PDAC and nanoparticle therapy, we designed dual-purpose functional theranostic probes comprising of dextran-coated SPIONs, which can be detected by MRI through strong shortening effects under longitudinal relaxation $\left(T_{1}\right)$ and transverse relaxation $\left(\mathrm{T}_{2}\right)$. In a modular construction system, these SPIONs were further conjugated with streptavidin (StAv-SPIONs). We coupled biotin-conjugated EPPT1, ${ }^{18}$ a synthetic nonimmunogenic peptide sequence targeted to uMUC1, to induce tumour-specific uptake of StAv-SPIONs. uMUC1 is a hallmark of early pancreatic tumorigenesis and is abundantly and ubiquitously expressed in PDAC. ${ }^{19}{ }^{20}$ Furthermore, biotin-conjugated MPAP were coupled to facilitate translocation of the complex into the cytosol. ${ }^{21}$ In addition, we linked biotin-conjugated siPLK1 to StAv-SPIONs, which resulted in the triple-labelled nanoparticle complex targeting PLK1. We estimate that siPLK1-StAv-SPIONs may carry approximately five siPLK1 per nanoparticle (figure $1 \mathrm{~A}$ and online supplementary table S1). TEM revealed an average core diameter of iron oxide nanoparticle core of $9.81 \pm 3.73 \mathrm{~nm}$ and a final siPLK1-StAv-SPIONs diameter of $123 \pm 14 \mathrm{~nm}$ (see online supplementary figure S1a,b). siPLK1-StAv-SPIONs showed surface modifications and change in particle height depicting conjugation of siPLK1, MPAP and EPPT1 compared with SPIONs and StAv-SPIONs as evident by atomic force microscopy imaging (see online supplementary figure S2). siPLK1 exhibited an entrapment efficiency of around $30 \%$ to StAv-SPIONs (see online supplementary table S1). In silico docking of biotinylated siPLK1 with streptavidin on the surface of StAv-SPIONs showed the siPLK1 residue to be protruding out from the streptavidin surface and not entrapped in the coating (see online supplementary figure S3). We detected accumulation of siPLK1-StAv-SPIONs in tumour cells as evident by a brown pellet (see online supplementary figure S4). Uptake and efficiency of siPLK1-StAv-SPIONs was shown by a significant increase of intracellular iron $\left(\mathrm{Fe}^{2+}\right)$ after 2 and $4 \mathrm{~h}$ in the murine PDAC cell line 6606PDA (figure 1B). We fully characterised the triple-labelled siPLK1-StAv-SPIONs for their composition, physical and physiochemical properties (see online supplementary tables S1-S3 and figures S1-S5).

In order to evaluate efficiency of siPLK1-StAv-SPIONs in silencing PLK1, we treated 6606PDA cells with siPLK1-StAv-SPIONs and evaluated the decrease in PLK1 expression over a period of $24 \mathrm{~h}$. We found a marked reduction in PLK1 expression on siPLK1-StAv-SPIONs treatment accompanied by accumulation of siPLK1-StAv-SPIONs in tumour cells detected by Perl's iron staining (figure 1C,D). We expected treatment efficacy of our triple-labelled siPLK1-StAv-SPIONs based on the ability of PLK1 suppression to prevent tumour cell proliferation by arresting the cells in the G2/M phase of mitosis. $^{7} 22223$ siPLK1-StAv-SPIONs treatment resulted in a pronounced increase in the number of cells in $\mathrm{G}_{2} / \mathrm{M}$ phase comparable to the selective PLK1 inhibitor, BI6727, employed in phase I and II cancer trials ${ }^{24}$ and proved to be efficient in silencing of PLK1 in tumour cells (figure 1E). To further confirm the silencing efficacy of siPLK1-StAv-SPIONs, we performed BrdU proliferation assay and found a significant suppression of proliferation in 6606PDA cells over a period of $48 \mathrm{~h}$ (figure 1F). Our delivery strategy allowed us to achieve substantial silencing of PLK1 overexpressed during the progression of PDAC. Interestingly, siPLK1-StAv-SPIONs showed lower cell toxicity and less generation of reactive oxygen species if compared with StAv-SPIONS lacking an active bioligand (see online supplementary figure S6). On evaluation of the migratory potential after siPLK1-StAv-SPIONs treatment, we found significant reduction in migration of cancer cells over a period of $48 \mathrm{~h}$ (see online supplementary figure S7). One of the apprehensions with regard to siPLK1 delivery is rapid inactivation in solution by nucleases. To answer this question, we performed in-solution stability studies in saline and 5\% faetal calf serum (FCS), which mimic physiological solutions and found marked protection for siPLK1 in siPLK1-StAv-SPIONs (see online supplementary figure S8). The aforementioned results show the successful delivery, functional efficiency and tolerance of siPLK1-StAv-SPIONs in vitro. Overall, our in silico data as well as in vitro data further confirmed that a nanoparticle-based siRNA delivery increases stability, accumulation of siPLK1-StAv-SPIONs and target specificity of siRNAs.

\section{Specificity and uptake mechanism}

We subsequently assessed the uptake specificity and uptake mechanism of siPLK1-StAv-SPIONs. We performed time-lapse live cell imaging with Cy5-labelled siPLK1-StAv-SPIONs with and without MPAP and EPPT1 peptide residues to monitor the siPLK1-StAv-SPIONs uptake. We found significant uptake of MPAP(+)EPPT1(+)siPLK1-StAv-SPIONs in cancer cells starting as early as $5 \mathrm{~min}$, while MPAP(-)EPPT1(-)siPLK1-StAv-SPIONs were not taken up during a period of $25 \mathrm{~min}$ (figure $2 \mathrm{~A}$ ). Quantitative analysis of Cy 5 mean fluorescent intensity on live cell imaging and FACS analysis showed a significant increase in MPAP(+)EPPT1(+)siPLK1-StAv-SPIONs compared with the respective controls without peptide ligands (figure $2 \mathrm{~B}$ and online supplementary figures S9a-S10a). We validated the importance of MPAP and EPPT1 for tumour-specific uptake measuring intracellular tumour iron content $6 \mathrm{~h}$ after siPLK1-StAv-SPIONs treatment in a syngeneic orthotopic tumour model. MPAP and EPPT1-conjugated siPLK1-StAv-SPIONs showed significant iron overload in tumours if compared with the respective control (figure 2C). Pretreatment with a monoclonal anti-MUC1 antibody resulted in an anti-MUC1 antibody concentration-dependent decrease in the uptake of Cy5-labelled siPLK1-StAv-SPIONs (figure 2D and online supplementary figures S9b-S10b). To assess the possible functional mechanism of uptake in cells, we hypothesised endocytosis as the possible mechanism for the uptake considering the size, particle charge and physical characteristics, which determine the chances for passive diffusion or active transport of the SPIONs. ${ }^{25-27}$ To further validate this hypothesis, we treated 6606PDA cells with the clathrin-dependent endocytosis inhibitor, Dynasore, ${ }^{28}$ prior to siPLK1-StAv-SPION incubation. Endocytosis inhibition diminished siPLK1-StAv-SPIONs uptake (figure 2E and online supplementary figure S9c) as confirmed by flow cytometric analysis (see online supplementary figure S10c). Dynasore blocked the uptake of siPLK1-StAv-SPIONs. To substantiate our finding, we evaluated the subcellular 
A

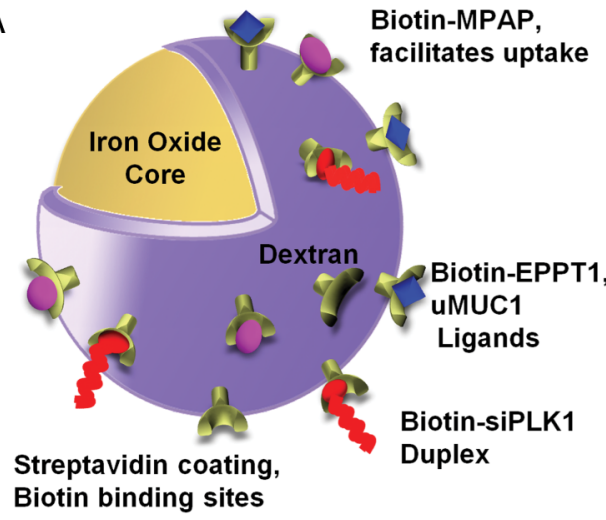

Streptavidin coating
Biotin binding sites

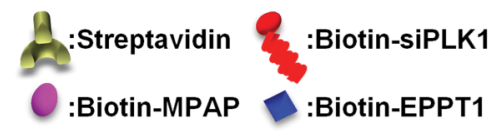

C
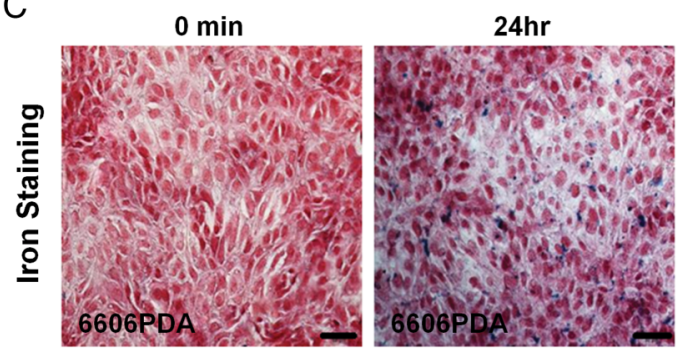

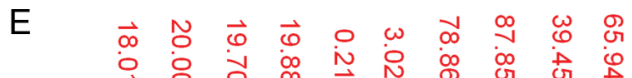

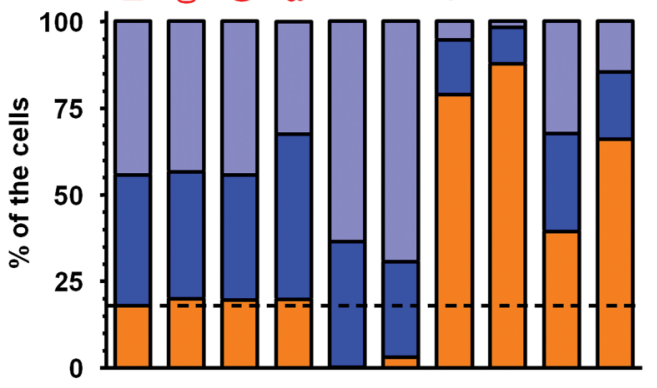

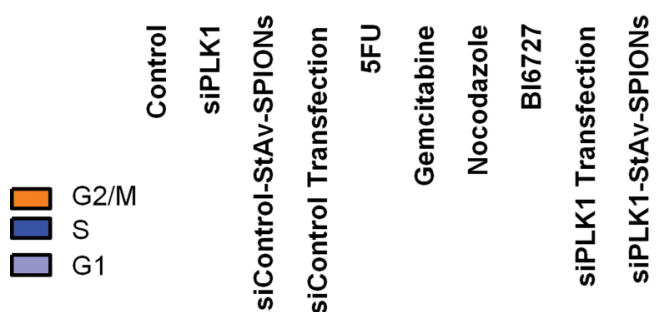

D

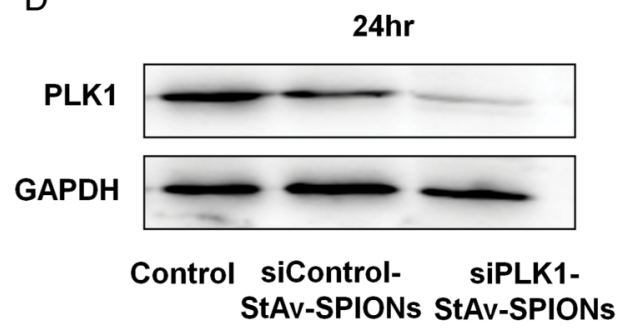

B

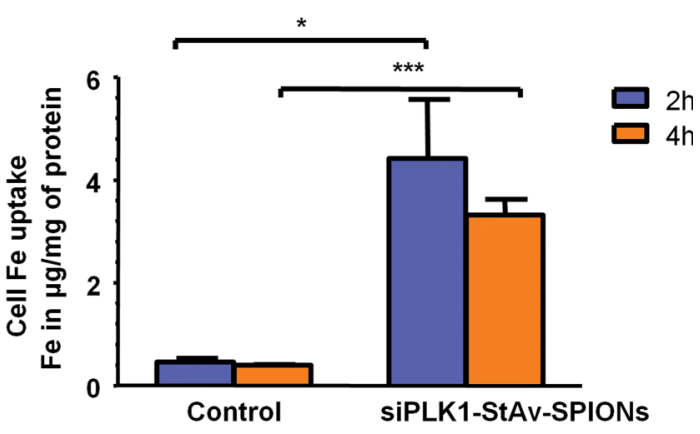

F

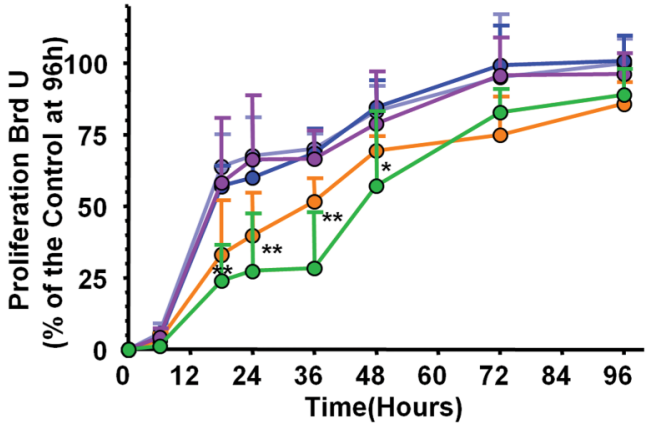

-o Control

-o siControl-StAv-SPIONs

-o- siPLK1-StAv-SPIONs

-o siControl Transfection

-o- siPLK1 Transfection

Figure 1 siPLK1-StAv-superparamagnetic iron oxide nanoparticles (SPIONs) are efficacious in silencing PLK1 and reducing tumour cell proliferation in vitro. (A) Schematic representation of siPLK1-coupled streptavidin-conjugated dextran-coated SPIONs (siPLK1-StAv-SPIONs) conjugated to the membrane translocation peptide for mediating transportation to the cytoplasm (myristoylated polyarginine peptides (MPAP)), the underglycosylated MUC1 (uMUC1)-specific peptide (EPPT1) and siRNA molecules targeting PLK1 (siPLK1). (B) Quantitative analysis of the uptake of siPLK1-StAv-SPIONs into tumour cells was estimated by iron content in cell lysates and showed significant uptake of iron in cells on 2 and $4 \mathrm{~h}$ incubation. Data represent mean $\pm S D(n=3$, Mann-Whitney $U$ test, $U=0.00, p=0.03(2 h), U=0.00, p=0.003(4 h)$ ). (C) Representative images showing uptake of siPLK1-StAv-SPIONs inside 6606PDA tumour cells at 0 and $24 \mathrm{~h}$ after incubation with siPLK1-StAv-SPIONs. (D) Immunoblotting of PLK1 revealed a decrease in PLK1 expression after siPLK1-StAv-SPIONs treatment $24 \mathrm{~h}$ after siPLK1-StAv-SPIONs treatment corresponding to mismatch control RNAi. (E) The silencing effect of PLK1 by siPLK1-StAv-SPIONs was manifested as the change in the cell cycle of diploid cells analysed using fluorescence activated cell sorting (FACS) analysis. siPLK1-StAv-SPIONs treatment showed stagnancy of the cells in G2/M phase of cell cycle ( 66\%) compared with that of control ( 18\%). 5-fluorouracil (5-FU) (G1 stagnancy), gemcitabine (G1 stagnancy), nocodazole (G2/M stagnancy) and BI6727 (specific PLK1 small-molecule inhibitor, G2/M stagnancy) served as controls for respective phases of cell cycle. (F) Quantitative estimation of BrdU-positive cell proliferation over a period of $48 \mathrm{~h}$ showed a significant decrease in proliferation of the murine pancreatic cancer cell line 6606PDA on treatment of siPLK1-StAv-SPIONs. Data represent mean $\pm S D(n=7-8$, Mann-Whitney U test. $U=21.00, p=0.27(6 h), U=6.00, p=0.004(18 h), U=4.00, p=0.002$ (24 h), $U=1.00, p=0.0003(36 h), U=7.50, p=0.02(48 h), U=15.00, p=0.08(72 h), U=8.00, p=0.06(96 h)),{ }^{* * *} p<0.001,{ }^{* *} p<0.01,{ }^{*} p<0.05$. 

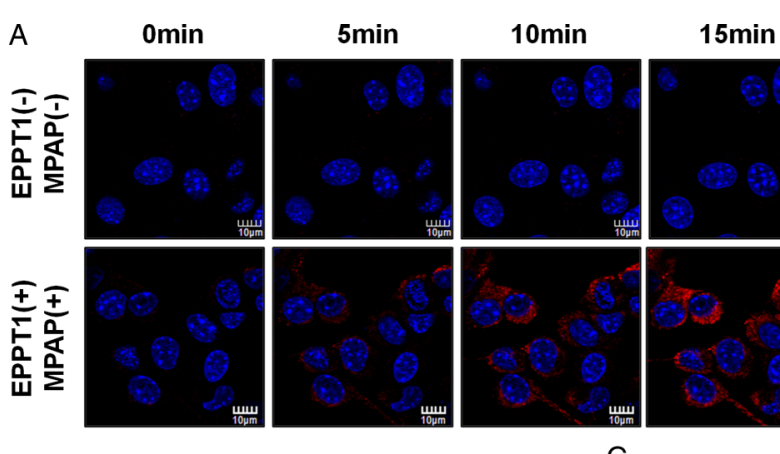

$20 \mathrm{~min}$
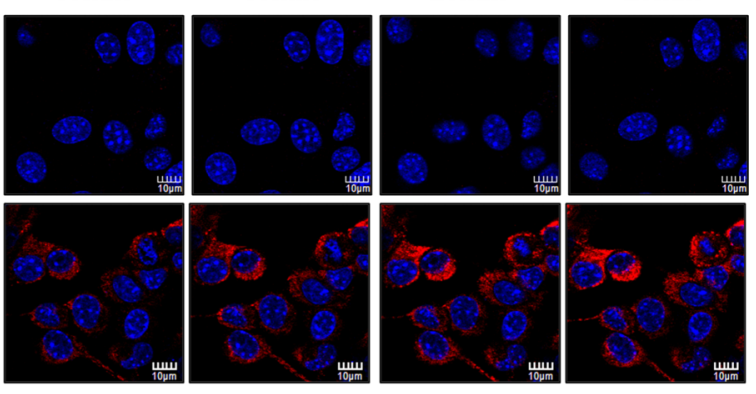

C
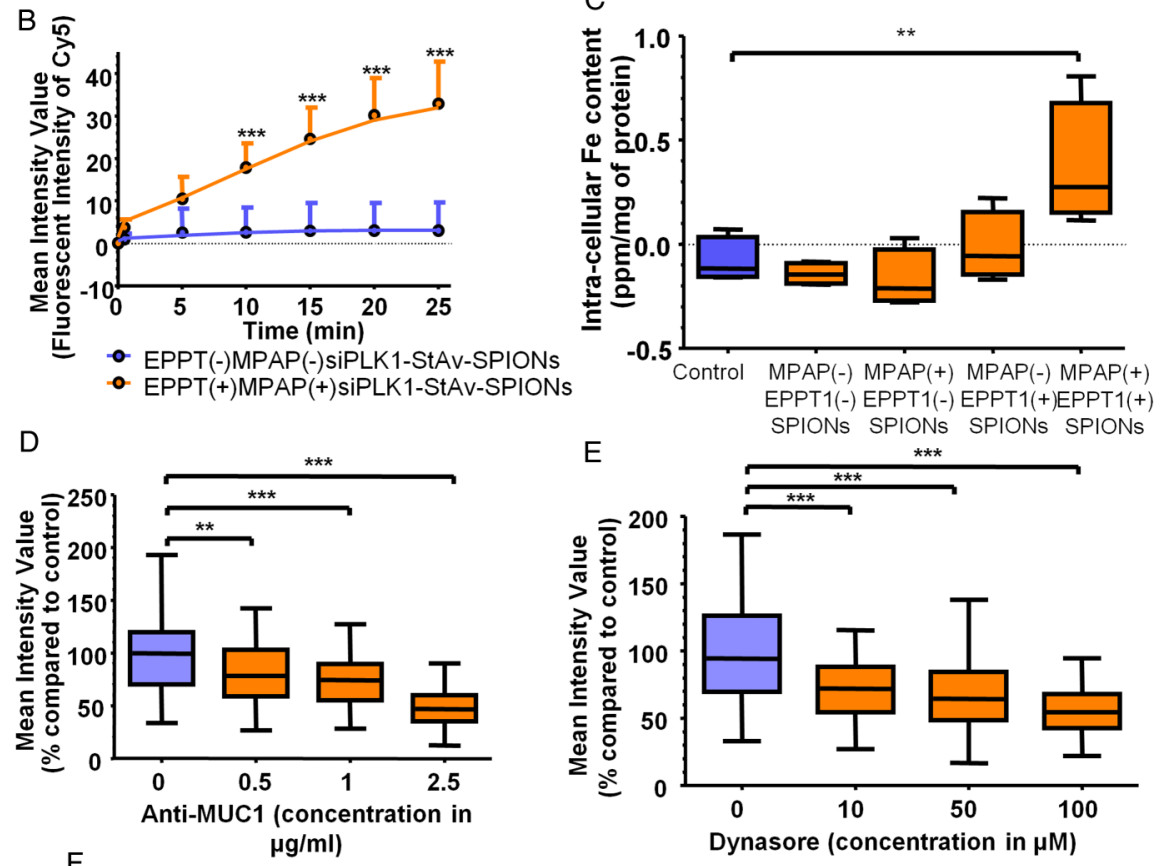

E

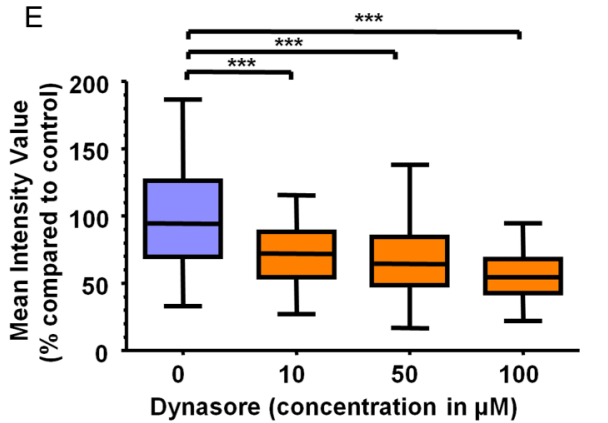

$30 \mathrm{~min}$
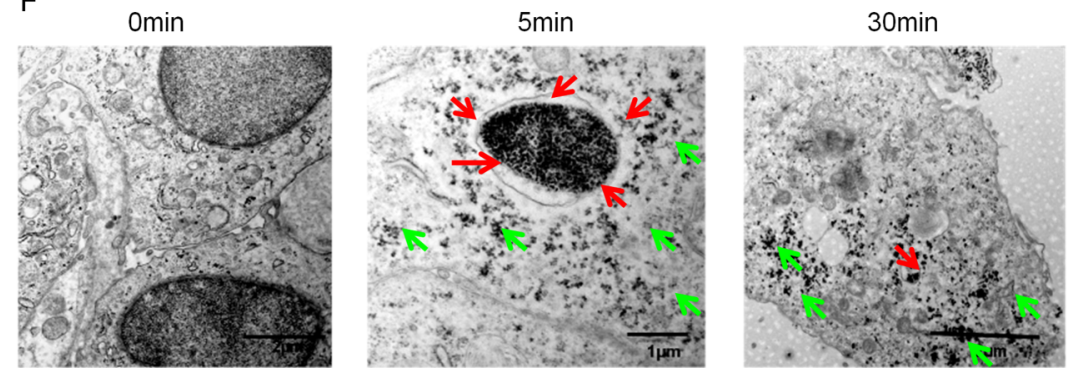

Figure 2 Uptake of siPLK1-StAv-superparamagnetic iron oxide nanoparticles (SPIONs) is tumour specific and driven by clathrin-dependent endocytosis. siPLK1-StAv-SPIONs were further conjugated with biotinylated Cy5 to analyse particle uptake using fluorescence microscopy. (A) Confocal microscopy showed accumulation of siPLK1-StAv-SPIONs coupled to EPPT1 and myristoylated polyarginine peptides (MPAP) (MPAP(+) EPPT1(+)siPLK1-StAv-SPIONs) in 6606PDA cells over the time period of $25 \mathrm{~min}$. In contrast, for siPLK1-StAv-SPIONs without EPPT1 and MPAP (MPAP (-)EPPT1(-)SPIONs), we did not detect uptake of nanoparticles over the time period of 25 min. Red fluorescence depicts nanoparticles (Cy5) and blue depicts nuclei (DAPI); scale bar=10 $\mu \mathrm{m}$. (B) Quantification of confocal images recorded over the time period of 25 min. siPLK1-StAv-SPIONs uptake was quantified as mean fluorescence intensity of Cy5. Data are expressed as mean \pm SD. Mann-Whitney $U$-test. $U=13.00, p=0.0003(5$ min), $\mathrm{U}=9.00, p=0.0001$ (10 min), $U=6.00, p<0.0001$ (15 min), $U=4.00, p<0.0001$ (20 min), $U=3.00, p<0.0001$ (25 min). (C) Box and whisker diagram show intracellular iron concentration in tumours harvested $6 \mathrm{~h}$ after MPAP(+)EPPT1(+) siPLK1-StAv-SPIONs treatment as well as the influence of MPAP - and EPPT1 - on the uptake of siPLK1-StAv-SPIONs. MPAP and EPPT1 are both necessary for the uptake of siPLK1-StAv-SPIONs by tumour cells. Kruskal-Wallis test followed by Dunn's multiple comparison post hoc test $H=11.89, p=0.01$. (D) Quantification of mean intensity value of Cy5 in relation to anti-MUC1 antibody concentration blocking the uptake of siPLK1-StAv-SPIONs ( $n=80-100$ cells from four different experiments). Kruskal-Wallis test followed by Dunn's multiple comparison post hoc test. $H=110.1, p<0.0001$. (E) Dynasore, an inhibitor of clathrin-dependent endocytosis, resulted concentration dependent in a decreased uptake of siPLK1-StAv-SPIONs. Quantification of mean intensity value of Cy5 corresponding to Dynasore-dependent reduction of SPION uptake ( $n=80-100$ cells from four individual experiments). Kruskal-Wallis test followed by Dunn's multiple comparison post hoc test $H=86.47, p<0.0001$. (F) Representative images of transmission electron microscopy revealed absent SPIONs at 0 min and uptake of siPLK1-StAv-SPIONs into the endosomal compartment (red arrow) as early as 5 min. After 30 min, most of the SiPLK1-StAv-SPIONs are detected in the cytoplasm (green arrows). The release from endosomal compartment to cytoplasm is believed to be due to the proton sponge effect. ${ }^{* * *} p<0.001,{ }^{* *} p<0.01,{ }^{*} p<0.05$. 
localisation of siPLK1-StAv-SPIONs on TEM, where we detected accumulation of siPLK1-StAv-SPIONs in membraneconfined vacuoles as early as $5 \mathrm{~min}$ after siPLK1-StAv-SPIONs treatment (figure $2 \mathrm{~F}$ ) while pronounced cytoplasmic localisation became only apparent at later time points. We correlated these findings to immunofluorescence staining for EEA1. Co-localisation with the early endosomal marker, EEA1, over a period of $30 \mathrm{~min}$ revealed that siPLK1-StAv-SPIONs are incompletely $(\sim 20 \%)$ co-localised with EEA1 and spread to the cytosol at later time points (see online supplementary figure $\mathrm{S} 11)$. In order to achieve successful gene silencing, siRNA has to interact with the RNA-induced silencing complex (RISC). Perinuclear cytoplasmic localisation of siRNA enhances the potential to interact with RISC. ${ }^{29-31}$ Frequent cytoplasmic localisation during later time points may suggest an escape of siPLK1-StAv-SPIONs from the membrane-confined vacuoles, which possibly represent endosomes to the cytoplasm (see online supplementary figure S12).

\section{MRI in tumour-bearing mice}

To be of therapeutic relevance, siPLK1-StAv-SPIONs need to be delivered specifically to the site of the tumour. To monitor the delivery of siPLK1-StAv-SPIONs to the tumour, we employed MRI in mice-bearing syngeneic orthotopic tumours before and $6 \mathrm{~h}$ after siPLK1-StAv-SPIONs intravenous injection. siPLK1-StAv-SPIONs possessed predominant proton relaxation rates of the surrounding water proton spins, which was associated with the magnetic susceptibility of the particles leading to $T_{2}$ relaxation, giving rise to signal reduction on $\mathrm{T}_{2}$-weighted images. ${ }^{32}$ As per our anticipation, siPLK1-StAv-SPIONs induced significant diminution in $\mathrm{T}_{2}$ relaxivity as evident by a decrease in contrast in the tumour compared with the tumour before siPLK1-StAv-SPIONs treatment $6 \mathrm{~h}$ after the application (figure 3A). Pseudo-colour mapping of MRI images showed significant reduction in tumour intensity in the region of interest (ROI) $6 \mathrm{~h}$ after siPLK1-StAv-SPIONs treatment (figure 3B). Furthermore, MRI showed a distinct decrease in contrast with a greater number of pixels in the tumour ROI with less intensity $6 \mathrm{~h}$ after siPLK1-StAv-SPIONs treatment. This was further verified by relative comparison of contrast of the number of pixels related to lesser intensity before injection (figure 3C). We further validated the accumulation of the siPLK1-StAv-SPIONs in tumour using quantitative analysis of iron overload calculated by $\mathrm{R}_{2}$ * single-peak fat-corrected echo images. $\mathrm{R}_{2}{ }^{*}$ value over $100 \mathrm{~s}^{-1}$ was considered to be a surplus of iron in the tissue. ${ }^{33}$ siPLK1-StAv-SPIONs-treated animals showed a significant increment in $\mathrm{R}_{2} *$ values if compared with control without any SPION treatment (figure 3D). To demonstrate the imaging capabilities of siPLK1-StAv-SPIONs and the importance of MPAP and EPPT1 for the tumour-specific uptake in a syngeneic orthotopic tumour model, we imaged the animals $6 \mathrm{~h}$ after injection of siPLK1-StAv-SPIONs with and without MPAP and EPPT1. Though there was uptake into the tumour with MPAP or EPPT1 as single ligands bound to SPIONs, the combination of MPAP and EPPT1 provided maximal uptake by the tumour. Thus, MPAP and EPPT1 are equally necessary for siPLK1-StAv-SPIONs tumour-specific uptake (figure $3 \mathrm{E}$ ). We confirmed our finding of the importance and specificity of MPAP and EPPT1 for our probes to accumulate in tumour harvested from the syngeneic orthotopic tumour model using Perl's iron staining (figure 3F). We believe EPPT1 drives the specificity of siPLK1-StAv-SPIONs towards tumour, whereas MPAP acts in the process of internalisation and endosomal escape. Furthermore, we confirmed accumulation of siPLK1-StAv-SPIONs using Perl's iron staining in tumours harvested $6 \mathrm{~h}$ after intravenous application of siPLK1-StAv-SPIONs.
Staining images showed marked accumulation of siPLK1-StAv-SPIONs (blue) in the tumour tissue (figure 3G). Taken together, these results demonstrate significant delivery of siPLK1-StAv-SPIONs to the tumour in vivo, which can be quantified by MRI.

\section{Therapeutic efficacy of siPLK1-StAv-SPIONs in a murine syngeneic orthotopic pancreatic cancer model}

To address the therapeutic efficiency of siPLK1-StAv-SPIONs, we performed a randomised, placebo-controlled animal study employing a murine syngeneic orthotopic pancreatic adenocarcinoma model. Treatment was started 14 days after tumour inoculation. We detected a significant decrease in the harvested tumour volume from $49 \pm 6 \mathrm{~mm}^{3}$ to $53 \pm 11 \mathrm{~mm}^{3}$ after 4 weeks of siPLK1-StAv-SPIONs treatment. To monitor the influence of siPLK1-StAv-SPIONs treatment on the tumour volume, we performed MRI at weekly intervals and analysed the tumour growth. Of note, siControl-StAv-SPIONs-treated group's tumour growth was not affected and increased from $57 \pm 7 \mathrm{~mm}^{3}$ to 303 $\pm 78 \mathrm{~mm}^{3}$ over the course of the experiment. In contrast, the siPLK1-StAv-SPIONs-treated group showed complete tumour growth arrest (figure 4A). Quantitative tumour volume analysis confirmed significant difference in tumour volumes over the duration of treatment on siPLK1-StAv-SPIONs treatment compared with the corresponding mismatch control-RNAi (figure 4B and online supplementary figure S13). Immunoblotting and quantification of PLK1 expression from harvested tumours showed significant silencing of PLK1 expression upon siPLK1-StAv-SPION treatment (figure 4C). siPLK1-StAv-SPIONs showed therapeutic efficacy in vivo via reducing proliferation (figure 4D), increasing apoptosis (figure 4E), increasing caspase 9 and caspase 3 activities (see online supplementary figure S14), eventually resulting in an increase in median survival (96 vs 76 days, $p=0.0005 . \chi_{1 \mathrm{DF}}^{2}$ $\mathrm{LR}_{\mathrm{C}}=12.01$ ) (figure $4 \mathrm{~F}$ and online supplementary figures $\mathrm{S} 15 \mathrm{~b}-\mathrm{d}$ and S16a-b). Small-molecule PLK1 inhibitor, BI6727 treatment, showed a dismal median survival of 21 days due to necrosis in gastric and duodenal mucosa as evident by Ki67 immunohistochemistry (see online supplementary figure S17f).

A major potential obstacle for the siPLK1-StAv-SPIONs treatment could be short-term serum bioavailability and nonspecific silencing of siPLK1 in non-tumour tissue that would lead to cellular toxicity, including hemotoxicity, as well as fatigue. We excluded any organ toxicity induced by siPLK1-StAv-SPIONs (see online supplementary tables S4-S6). siPLK1-StAv-SPIONs treatment did cause any of the adverse effects associated with PLK1 silencing via small-molecule inhibitors (see online supplementary table S6). We excluded a non-specific effect of siPLK1-StAv-SPIONs in silencing of siPLK1 through the aid of quantitative real-time PCR performed in different organs harvested after completion of treatment. We detected no significant downregulation in siPLK1 RNA expression in organs other than tumours of the siPLK1-StAv-SPIONs-treated group, indicating absence of cytotoxicity caused by siPLK1-StAv-SPIONs treatment (see online supplementary figure S18). A major concern related to the in vivo delivery of metallic nanoparticle delivery system is the rapid clearance of nanoparticles from the plasma, thus limiting its efficacy owing to lower plasma half-life. ${ }^{34}$ Considering a non-compartmental first-order kinetic model after siPLK1-StAv-SPIONs treatment, we determined the serum plasma half-life of siPLK1-StAv-SPIONs with approximately $210 \mathrm{~min}$ (see online supplementary figure S19 and table S7) with a mean residual time of approximately $292 \mathrm{~min}$. Thus, siPLK1-StAv-SPIONs present with a significant serum half-life for sufficient transfer to the tumour site, suggesting a prolonged 
A

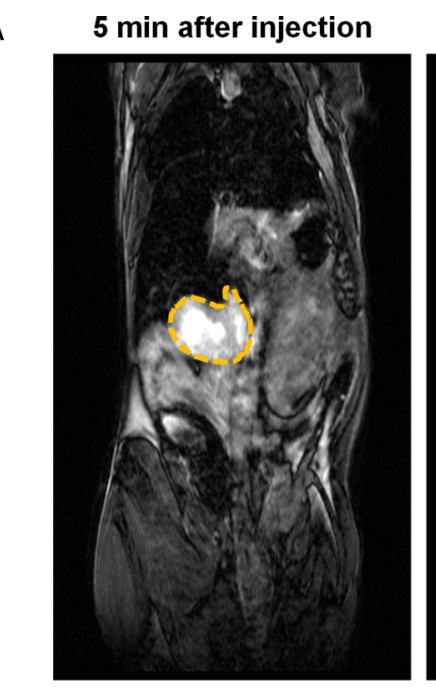

6 hours after Injection

B

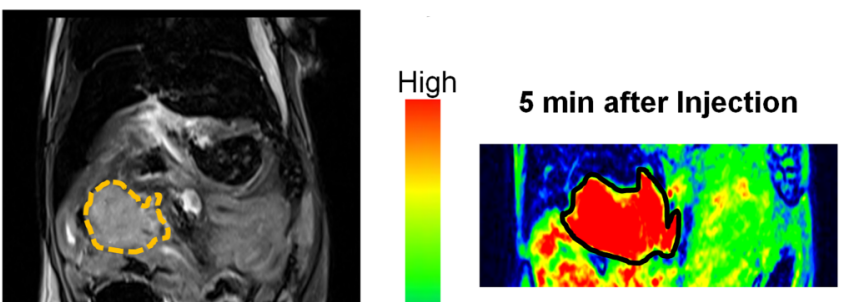

6 hours after Injection
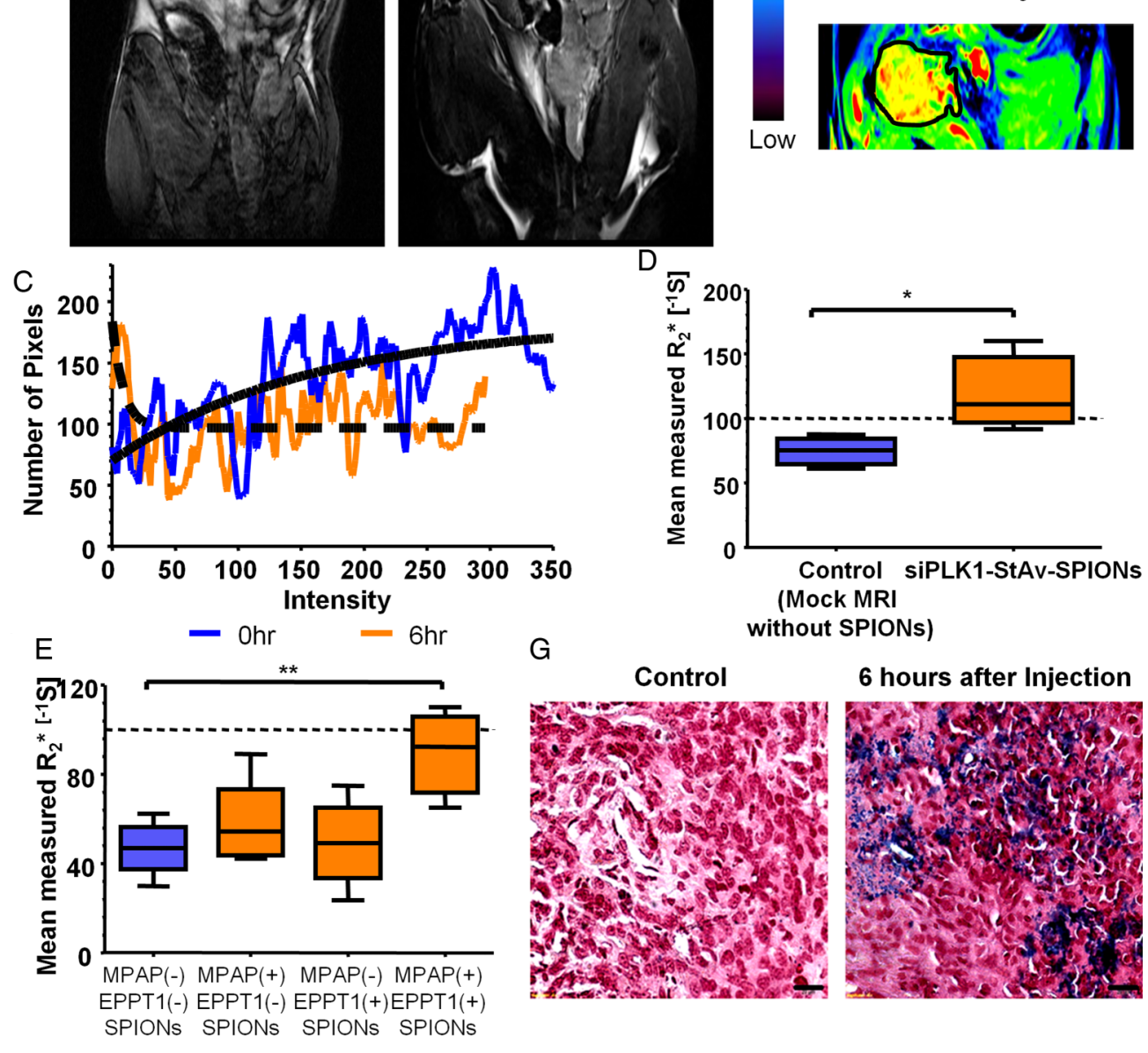

G

$\mathrm{D}$

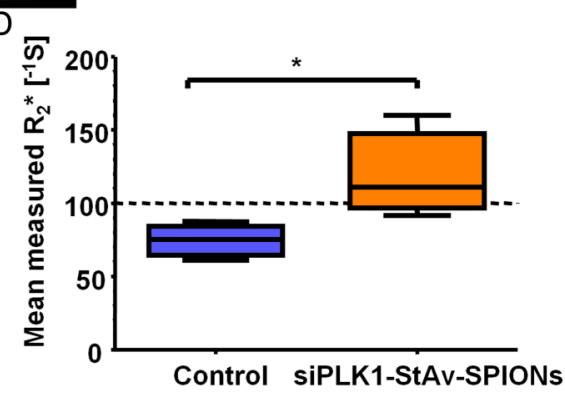

(Mock MRI

without SPIONs)
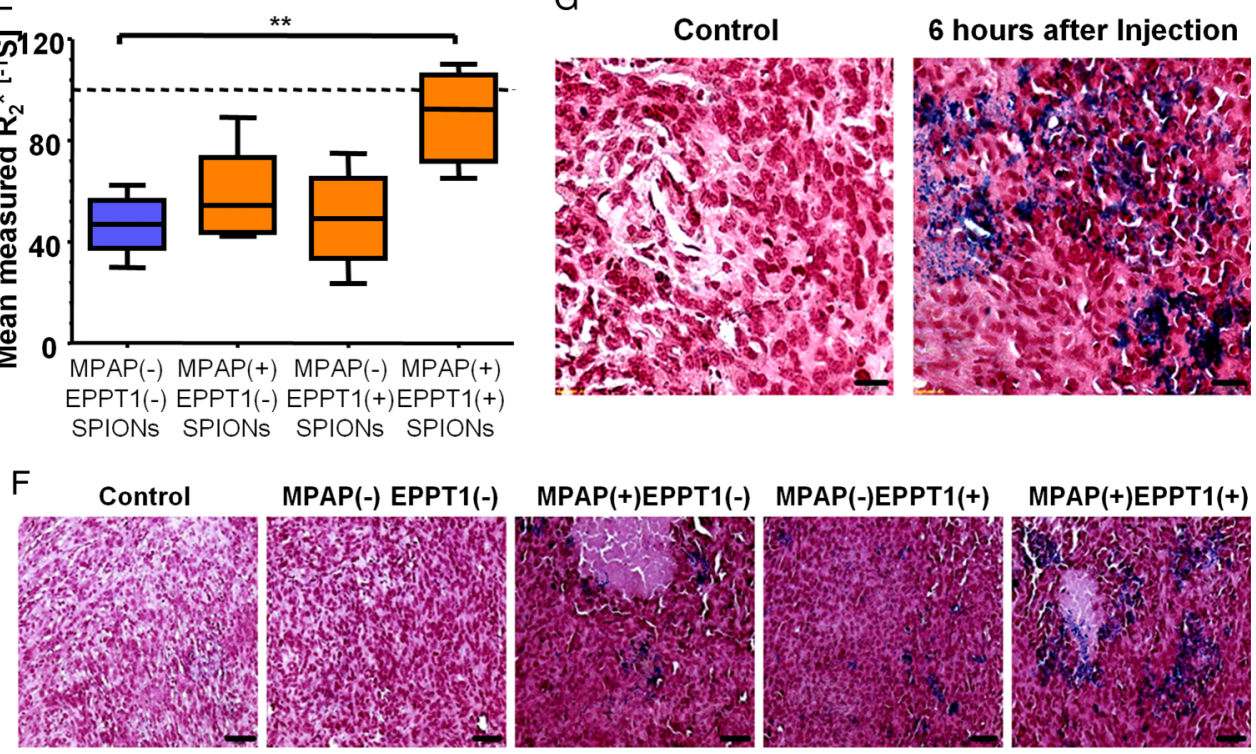

Figure 3 In vivo imaging of delivery of siPLK1-StAv-superparamagnetic iron oxide nanoparticle (SPIONs) to the tumour. (A) In vivo MRI was performed on the mice-bearing syngeneic orthotopic tumours before and $6 \mathrm{~h}$ after intravenous administration of siPLK1-StAv-SPIONs. After injection of siPLK1-StAv-SPIONs, there was reduction in $\mathrm{T}_{2}$ relaxivity in the tumours as evident by a decrease in contrast. (Dotted lines mark the periphery of tumour.) (B) Colour contrast images show reduction in $\mathrm{T}_{2}$ relaxivity compared with that before injection. (C) Region of interest analysis of the contrast of pixels related to respective intensity $6 \mathrm{~h}$ after siPLK1-StAv-SPIONs treatment. Black lines show a trend in the decrease in intensity/pixels $6 \mathrm{~h}$ after treatment (dotted) in comparison to baseline (solid line). (D) MRI on tumour-bearing mice was performed before and $2 \mathrm{~h}$ after intravenous administration of siPLK1-StAv-SPIONs. Quantitative analysis of iron uptake in tumours calculated by $\mathrm{R}_{2}{ }^{*}$ fat-corrected single-peak echo images. siPLK1-StAv-SPIONs-treated animals showed a significant iron overload in the tumour $(n=4-5$, Mann-Whitney $U$ test. $U=0.00, p=0.015)$. Control animals without siPLK1-StAv-SPIONs treatment did not show iron overload. (E) Box and whisker diagram show the influence of myristoylated polyarginine peptides (MPAP) and EPPT1 on iron overload in tumour calculated using quantitative analysis of $R_{2}{ }^{*}$ fat-corrected single-peak echo images. MPAP(+)EPPT1(+) siPLK1-StAv-SPIONs-treated animals showed significant iron overload. Kruskal-Wallis test followed by Dunn's multiple comparison post hoc test. $\mathrm{H}=8.24, \mathrm{p}=0.007$. (F) Perl's iron staining in tumours $6 \mathrm{~h}$ after injection indicated presence of siPLK1-StAv-SPIONs inside the tumour (blue), confirming delivery of siPLK1-StAv-SPION to the tumour. Scale bar=20 $\mu \mathrm{m}$. (G) Representative images of Perl's iron staining underlining the dependency of uptake of siPLK1-StAv-SPIONs on both MPAP and EPPT1 ligands. ${ }^{*} p<0.01,{ }^{*} p<0.05$. 

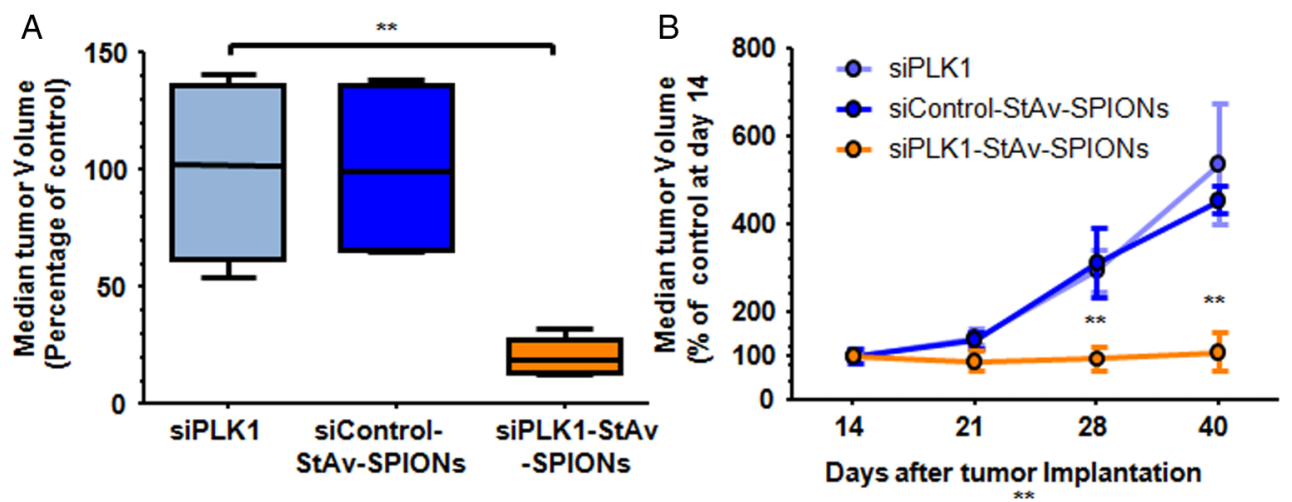

C
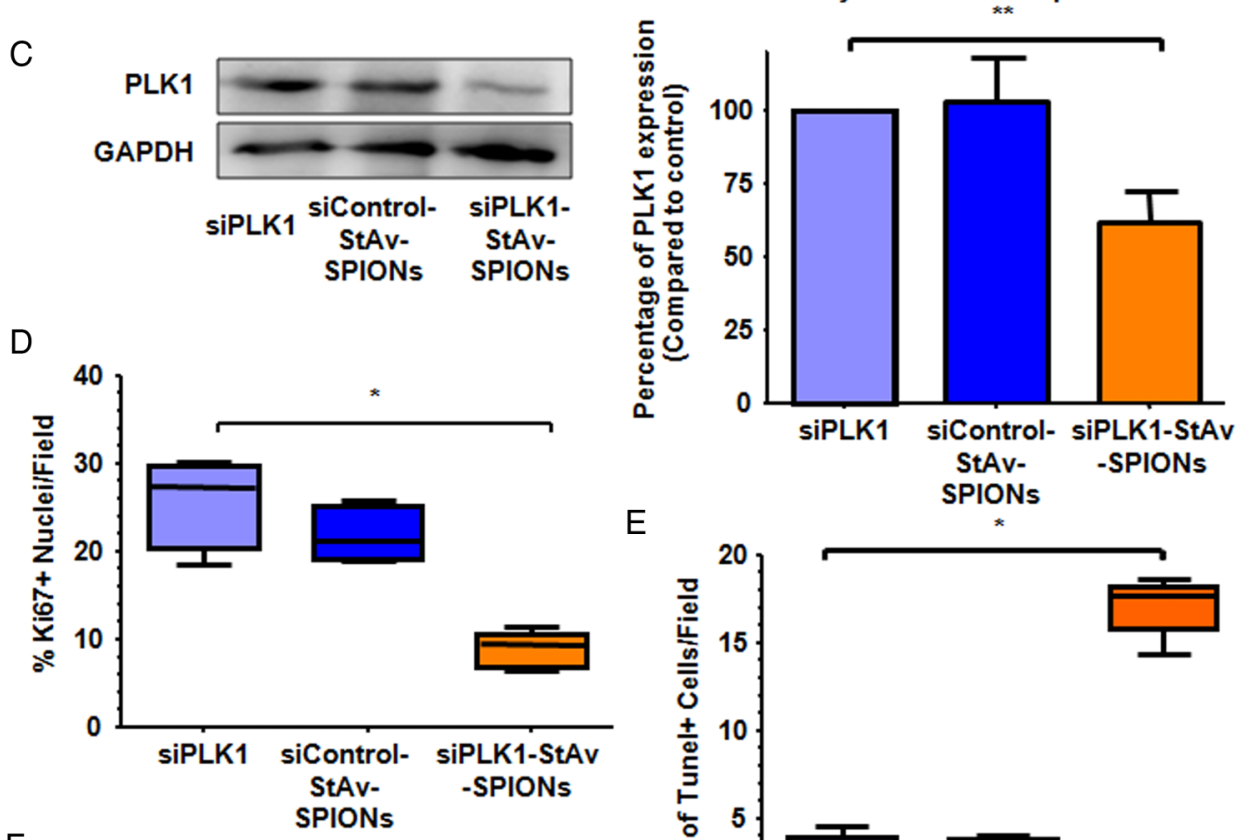

$\mathrm{F}$
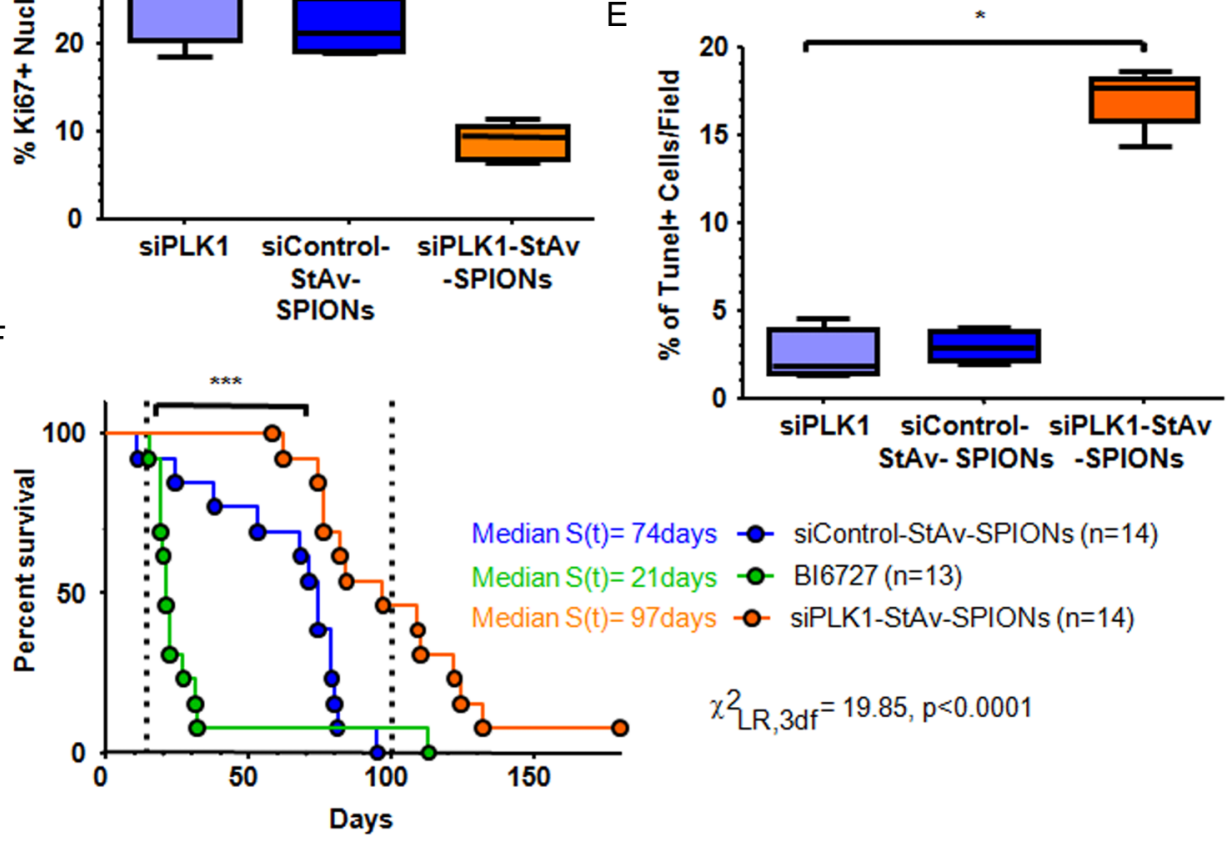

Figure 4 siPLK1-StAv-superparamagnetic iron oxide nanoparticle (SPIONs) arrest tumour progression in a syngeneic orthotopic tumour model. Tumour-bearing mice were injected intravenously with either siPLK1-StAv-SPIONs, naked siPLK1 or mismatch control siRNA-StAv-SPIONs 14 days after tumour implantation for $3 q D$ over a time period of 4 weeks. (A) Median tumour volume after completion of therapy shows significant reduction in tumour volume of siPLK1-StAv-SPION-treated animals compared with either naked siPLK1 or mismatch-control-siRNA-StAv-SPION ( $n=5$, KruskalWallis test followed by Dunn's multiple comparison post hoc test. $\mathrm{H}=0.67, \mathrm{p}=0.003$ ). (B) In situ tumour measurement using MRI showed stagnancy of tumour growth in the siPLK1-StAv-SPION-treated group over the period of treatment. siControl-StAv-SPIONs or naked PLK1 siRNA did not show any effect on tumour volume ( $n=3-4$, Mann-Whitney $U$ test. $U=0.00, p=0.07$ (21 days), $U=0.00, p=0.002$ ( 28 days), $U=0.00, p=0.002$ (40 days)). (C) Immunoblotting of PLK1 revealed a decrease in PLK1 expression in the siPLK1-StAv-SPIONs-treated group. Quantification of the expression level showed a significant decrease in PLK1 expression. Data represent mean \pm SD of three individual experiments. Kruskal-Wallis test followed by Dunn's multiple comparison post hoc test. $H=9.91, p=0.007$. (D) Quantitative analysis of the percentage of Ki67-positive nuclei in tumour resection specimen proved a significant decrease in proliferation in the siPLK1-StAv-SPION-treated group ( $n=4-5$, Kruskal-Wallis test followed by Dunn's multiple comparison post hoc test. $\mathrm{H}=9.10, \mathrm{p}=0.01$ ). (E) Tunel assay showed a significant increase in apoptotic nuclei upon siPLK1-StAv-SPION treatment $(n=4-5$, Kruskal-Wallis test followed by Dunn's multiple comparison post hoc test. $H=9.09, p=0.01)$. (F) Kaplan-Meier survival analysis from the time of enrolment to treatment with siControl-StAv-SPIONs $(n=14), B I 6727(n=13)$ or siPLK1-StAv-SPIONs ( $n=14)$. Dotted line window indicates maximum duration of therapy. Median survival time of siPLK1-StAv-SPION treatment (96 days) was significantly different to the siControl-StAv-SPION treatment (74 days). ${ }^{* *} p<0.001,{ }^{* *} p<0.01,{ }^{*} p<0.05$. 
contact time, which might increase the specific binding to uMUC1 on tumour cells and subsequent uptake.

\section{Efficacy of siPLK1-StAv-SPIONs treatment in the endogenous murine pancreatic cancer model in KPC mice}

The orthotopic syngeneic tumour model has been previously criticised for a less developed tumour vasculature and tumour microenvironment compared with an endogenous tumour model. ${ }^{35}$ We thus employed a genetically engineered mouse model (GEMM) for pancreatic cancer in the KPC mice. We replicated a prospective, randomised, double-blind, placebocontrolled pilot trial in KPC mice to evaluate treatment efficacy of siPLK1-StAv-SPIONs on tumour progression in established tumours. We found a complete growth arrest 1 week after siPLK1-StAv-SPIONs treatment, whereas siControl-StAv-SPIONs did not have an effect on tumour progression (figure $5 \mathrm{~A}$ and online supplementary figure S20). Tumours harvested after 2 weeks of treatment showed a significant decrease in PLK1 mRNA and protein expression upon treatment with siPLK1-StAv-SPIONs (figure 5B, C). We observed 36\% $\pm 10 \%$ to $57 \% \pm 14 \%$ of area of tumour cells (well, moderate, poorly differentiated and necrosis) occupied with nanoparticles probes, whereas stroma and normal cells did not show prominent accumulation. $13 \% \pm 2 \%$ was considered as background (figure $5 \mathrm{D}$ and online supplementary figure S21). Quantitative assessment of Ki67 immunohistochemistry staining revealed a significant decrease in proliferation, cancer cells mitotic arrest as evident by significant accumulation of phospho-histone $\mathrm{H} 3$ ( $\mathrm{pHH} 3$ ) (figure 5E and online supplementary figure S22b, c) as well as an increase in apoptosis in tumours from mice treated with siPLK1-StAv-SPIONs (figure 5F and online supplementary figures S22d,e and S23a). PLK1 silencing induced mitotic arrest as evident by a marked increase in nuclear pericentrin and a decrease in nuclear translocation of $\operatorname{cdc} 25$ and CDK1. We observed synthetic lethality of $\mathrm{p} 53^{\mathrm{R} 172 \mathrm{H}}$ upon PLK1 silencing without any organ toxicity (see online supplementary figures S22f-i, S23b-e and table S8). We detected a significant increase in caspase 9 and caspase 3/7 activities on siPLK1-StAv-SPIONs treatment with minimal change in caspase 8 activity, confirming the fact that PLK1 silencing confers apoptosis by activating the mitochondrial dependent apoptotic cascade (see online supplementary figures S24-S25). Of interest, immunohistochemistry of tumour specimens treated with siPLK1-StAv-SPIONs showed absence of PLK1 expression only in tumour cells, whereas the surrounding stroma still stained positive for PLK1, indicating specificity of the SPIONs to cancer cells (figure 5G and online supplementary figure S26). Of note, we found significant accumulation of siPLK1-StAv-SPIONs only in MUC1-positive metastatic tissues, assuring siPLK1-StAv-SPIONs specificity towards MUC1 (see online supplementary figure S27).

Accumulation of siPLK1-StAv-SPIONs in tumour cells via EPPT1 and MPAP confers efficacy, which was evident by a decreased expression of PLK1 in tumour cells but not in the stromal compartment.

We observed complimentary findings in KPC mice to that of the syngeneic orthotopic tumour model in reducing tumour burden and silencing PLK1. The theranostic SPIONs presented in this study have largely overcome previous experimental and therapeutic limitations and form an effective treatment for experimental pancreatic cancer.

\section{DISCUSSION}

A characteristic feature in treatment failure of PDAC is the inability of traditional chemotherapies to reach the site of action. The goal of our study was to design and characterise functional dual-purpose SPIONs for non-invasive in vivo imaging as well as siRNA delivery for silencing of PLK1 in PDAC. Our results demonstrate a novel technology to deliver theranostics to PDAC using unique tumour-specific siPLK1-StAv-SPIONs. The tumour-directed accumulation is further substantiated using tumour-specific peptides conjugated to siPLK1-StAv-SPIONs. The synthesised multimodal probe demonstrated specificity towards an uMUC1 overexpressed on the tumour surface mediated by the peptide ligand EPPT1. The uMUC1-specific siPLK1-StAv-SPIONs probe introduced here can significantly advance our current ability to detect and treat PDAC. ${ }^{18}$ Conjugation of the peptide MPAP was used to increase the electrostatic interactions through the positively charged arginine motives and their hydrophobic interaction, which leads to direct translocation through the negatively charged glycocalyx. The addition of a myristoylated group facilitates insertion of peptides, proteins and other cargoes into a lipid bilayer due to direct hydrophobic interaction and allows the translocation of both positively and negatively charged peptides and their cargoes throughout the lipid bilayer of the plasma membrane either by endocytic or energy-independent uptake. $^{21} 36$

We expected treatment efficacy of our triple-labelled siPLK1-StAv-SPIONs based on the ability of PLK1 suppression to prevent tumour cell proliferation by arresting the cells in the $\mathrm{G}_{2} / \mathrm{M}$ phase of mitosis. ${ }^{722}$ Our delivery strategy allowed us to achieve substantial silencing of PLK1 overexpressed in PDAC and in turns its therapeutic intervention, resulting in reduced tumour proliferation comparable or superior to previously published results. ${ }^{7}$

We subsequently assessed the uptake specificity and possible uptake mechanism of siPLK1-StAv-SPIONs. Blocking of MUC1 resulted in a concentration-dependent decrease of the uptake of siPLK1-StAv-SPIONs pointing to the importance of the ligand EPPT1 specifically binding to uMUC1. To assess the possible functional mechanism of uptake in cells, we hypothesised endocytosis as the possible mechanism considering the size, particle charge and physical characteristics, which determine the chances for passive diffusion or active transport of the SPIONs. ${ }^{25-27} 37$ To further validate this hypothesis, we tested the effect of Dynasore pretreatment, an inhibitor of clathrin-dependent endocytosis, on siPLK1-StAv-SPIONs uptake. Dynasore blocked the uptake of siPLK1-StAv-SPIONs. In addition, siPLK1StAv-SPIONs showed partial co-localisation with the early endosomal marker, EEA1, over a period of $30 \mathrm{~min}$ confirming endosomal uptake. In order to achieve successful gene silencing, siRNA has to interact with the RISC. Perinuclear cytoplasmic localisation of siRNA enhances the potential to interact with RISC. Different mechanisms such as pore formation in the endosomal membrane, the proton sponge effect of protonable groups and fusion into the lipid bilayer of endosomes have been proposed to facilitate the endosomal escape in order to interact with RISC. Unsaturated amino groups of MPAP drive a proton sponge effect by sequestering proton pumps on endosomes, which causes endosomal rupture. Protonation induces an extensive inflow of ions and water into the endosomal environment, which subsequently leads to rupture of the endosomal membrane and release of the entrapped components. ${ }^{29}$ 38-40 Endosomal co-localisation of siPLK1-StAv-SPIONs at an early time point and more frequent cytoplasmic localisation during later time points suggested an endosomal escape mechanism most likely mediated by the proton sponge effect induced by MPAP. 
A

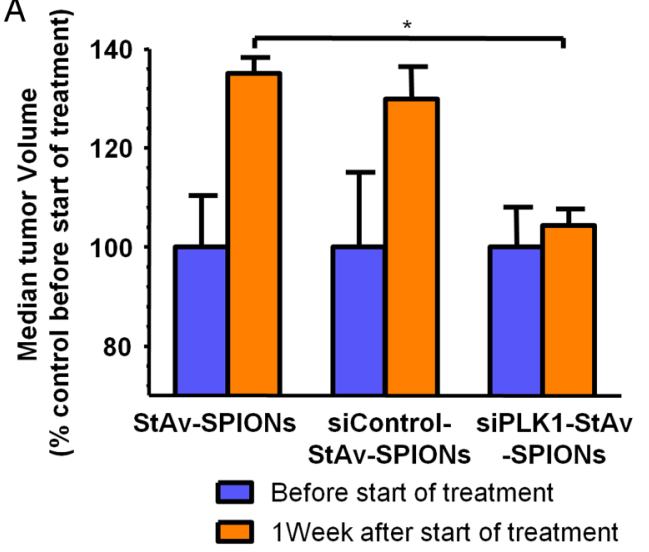

B

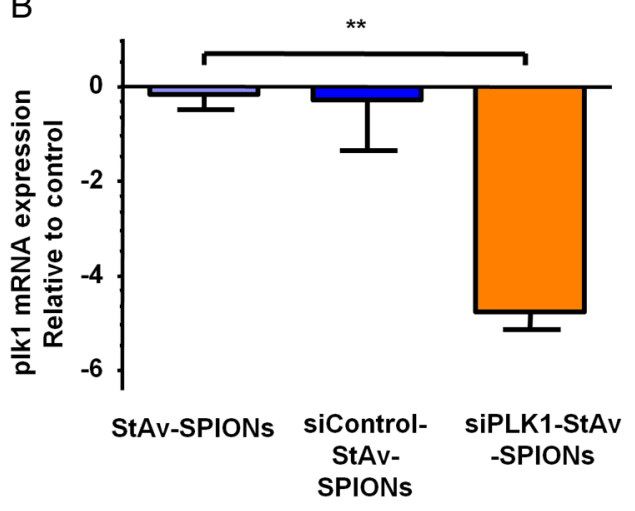

C

D StAv-SPIONs
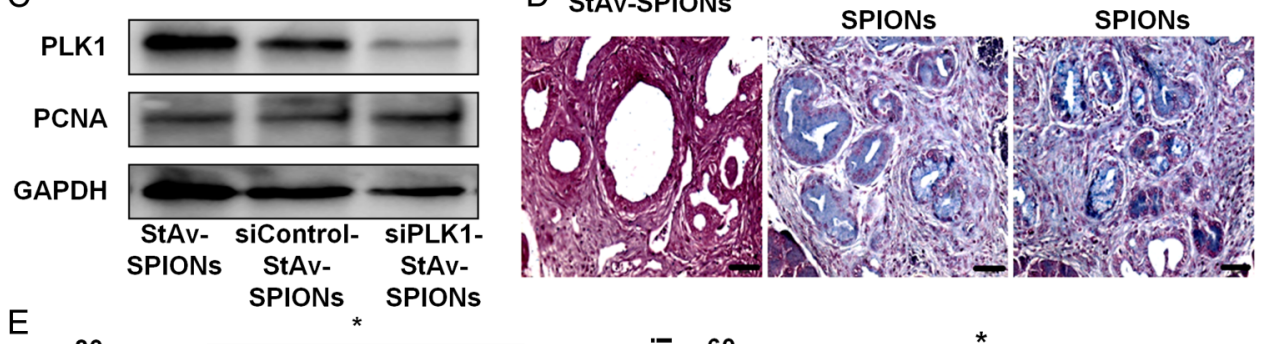

$E$
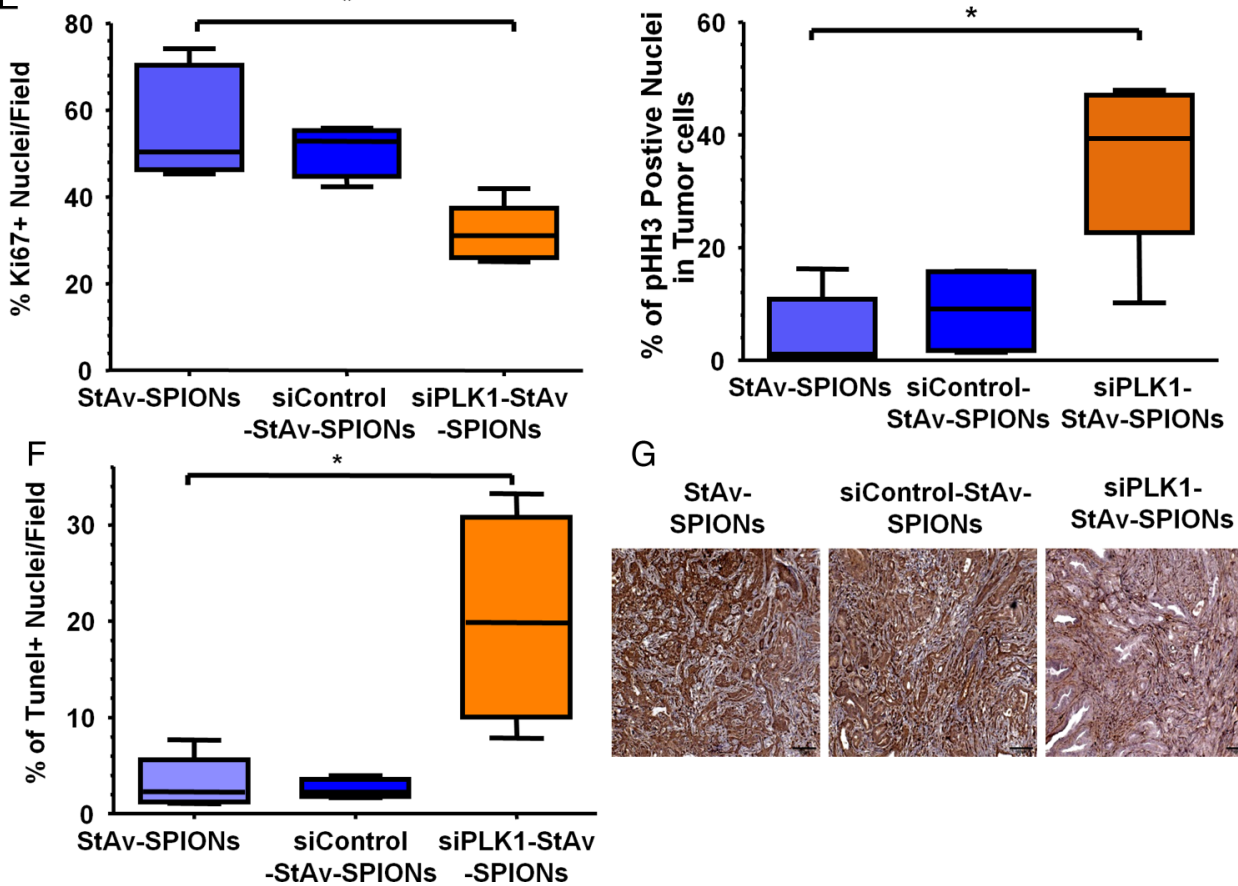

G

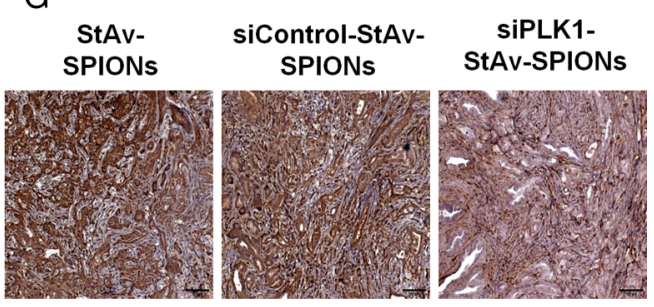

Figure 5 siPLK1-StAv-superparamagnetic iron oxide nanoparticle (SPIONs) prevent tumour progression in LSL-Kras ${ }^{\text {G12D }}$,LSL-Trp53 $3^{\mathrm{R} 172 \mathrm{H}}$, Pdx-1-Cre (KPC) mice. Tumour-bearing mice were randomised and (16-20 weeks old) were injected intravenously with either siPLK1-StAv-SPIONs, StAv-SPIONs or siControl-StAv-SPION for 3qD for two weeks. The investigator was blinded to the treatment. (A) Median tumour volume 1 week after start of therapy showed stagnancy in tumour growth in the siPLK1-StAv-SPIONs-treated animals compared with either StAv-SPIONs or siControl-StAv-SPIONs $(n=3-4)$. In situ tumour measurement using ultrasound showed significant stagnancy in tumour growth in the siPLK1-StAv-SPION-treated group over the period of treatment. siControl-StAv-SPIONs or StAv-SPIONs did not have any effect on tumour volume $(n=3-4$, Mann-Whitney $U$ test. U $=0.00$, $\mathrm{p}=0.02$ (after 1 week treatment)). (B and C) Quantitative real-time PCR and immunoblotting of PLK1 showed a decrease in PLK1 expression in the siPLK1-StAv-SPIONs-treated group. Quantitative analysis showed a significant decrease in PLK1 expression in respect to control. Data represent mean \pm SD of three individual experiments. Kruskal-Wallis test followed by Dunn's multiple comparison post hoc test. $H=22.00, p<0.01$. PCNA expression did not show any change. (D) Representative images of Perl's iron staining in tumours harvested after completion of treatment regimen indicated presence of siPLK1-StAv-SPIONs inside the tumour (blue), confirming delivery of siPLK1-StAv-SPION to the tumour. Scale bar=20 $\mu \mathrm{m}$. (E) Quantitative analysis of the percentage of Ki67-positive nuclei and phospho-histone $\mathrm{H} 3$ (pHH3) on immunohistochemistry on tumour sections revealed a significant decrease in proliferation in siPLK1-StAv-SPION-treated animals $(n=4-5$, Kruskal-Wallis test followed by Dunn's multiple comparison post hoc test. $\mathrm{H}=9.02, \mathrm{p}=0.01$ (Ki67), $\mathrm{H}=7.332, \mathrm{p}=0.02$ ( $\mathrm{pHH}$ )). (F) Tunel assay showed a significant increase in apoptotic Tunel-positive cells upon siPLK1-StAv-SPION treatment ( $n=4-5$, Kruskal-Wallis test followed by Dunn's multiple comparison post hoc test. $\mathrm{H}=7.74$, $\mathrm{p}=0.02)$. (G) Immunolabelling of PLK1 in tumour sections harvested from KPC mice upon treatment with StAv-SPIONs, siControl-StAv-SPIONs or siPLK1-StAv-SPIONs showed reduced expression of PLK1 only in the siPLK1-StAv-SPION-treated group and silenced expression was restricted to tumour cells. ${ }^{* *} \mathrm{p}<0.01,{ }^{*} \mathrm{p}<0.05$. 
Our study introduced the MRI of PDAC by tracking siPLK1-StAv-SPIONs. The added value in combining the siRNA delivery and imaging capabilities within a single nanoparticle allows monitoring of bioavailability and delivery can be directly correlated to the abundance of the therapeutic molecule at the site of the tumour or metastasis. Furthermore, SPIONs as contrast enhancer increase the sensitivity of MRI.

As PLK1 was previously identified as a promising treatment target in cancer, several PLK1 inhibitors have been developed by the pharmaceutical industry. There are now phase I studies for rigosertib and BI2536 available. ${ }^{41}{ }^{42}$ In the trial of BI2536, the typical toxicity of mitotic inhibitors, febrile neutropenia, was reported as the dose-limiting toxicity. This supports a need for targeted delivery as untargeted delivery of BI2536 to healthy tissue warranted dose reductions limiting its treatment efficacy. ${ }^{43}$

Therefore, targeted silencing of PLK1 by siRNA may offer several advantages over functional inhibition by small-molecule inhibitors. By its nature, siRNAs are highly specific and allow for the selective inhibition without affecting even closely related proteins compared with the relative promiscuity of kinase inhibitors. The biological response to protein depletion by siPLK1 can also differ from its functional inhibition by small molecules as protein deletion leads to the loss of both, kinase and polo-box functionality, upon PLK1 gene silencing. The duration of a drug effect with minimal side effects can be achieved by siPLK1 and is thus another attractive advantage. Once RNA inhibition is established within mammalian cells, gene silencing can persist for many days due to the relative stability of the activated RISC complex in the presence of its complementary mRNA while the duration of the treatment effect of smallmolecule inhibitors depends on the inhibitory mechanism. ${ }^{44}$

We found a drastic decrease in the tumour volume in syngeneic orthotopic tumour model upon siPLK1-StAv-SPIONs treatment, and these results prove that siPLK1-StAv-SPIONs are available at the tumour site and trigger RNAi-mediated silencing of PLK1 within solid tumours, silencing target expression at a magnitude sufficient to induce the mitotic disruption and apoptosis of tumour cells and thus reducing proliferation. siPLK1-StAv-SPIONs by arresting mitotic entry decrease proliferation and by activating mitochondrial-dependent apoptotic pathway and providing synthetic lethality to $\mathrm{p} 53^{\mathrm{R} 172 \mathrm{H}}$ increase apoptosis (see online supplementary figure S25).

There are recent studies reporting the use of EPPT1-coupled SPIONs in detection and monitoring tumour response to chemotherapy in subcutaneous xenografts of pancreatic cancer cell lines in immunodeficient nu/nu mice. ${ }^{18}{ }^{45}$ Similarly, Medarova et al ${ }^{21}$ elucidated the efficacy of siRNA delivery and in vivo imaging using MPAP-coupled SPIONs in subcutaneous xenografts of pancreatic cancer cell lines in immunodeficient nude mice. Although these studies provide a proof of principle for the use of EPPT1 and MPAP-targeted therapy, immunodeficient in vivo models used for the studies suffer from several shortcomings since tumour development and progression cannot be evaluated in the absence of a proper functioning immune system. One major drawback of xenografts is that they do not reproduce the primary site of the human cancer and are characterised by minimal genetic heterogeneity. ${ }^{46}$ Also, xenografts lack the architectural and cellular complexity of in vivo tumours, which include inflammatory cells, vascular components and other stromal components. These differences in tumour cells and the microenvironment are probably relevant for the therapeutic delivery and thus data from these models cannot be transferred to a clinical setting. ${ }^{47}$ To address these shortcomings, we employed GEMMs for pancreatic cancer. These KPC mice have been developed to provide in situ tumour development in an immunocompetent animal setting, addressing many of the shortcomings of cell-based assays and xenografts. KPC mice spontaneously develop PDAC that resemble the histopathological and molecular features of human PDAC and are regarded to be relevant for validating treatment efficacy in patients. ${ }^{48} 49$

We replicated pilot study in KPC mice to evaluate the efficacy of siPLK1-StAv-SPIONs on tumour progression. We observed similar findings to that of the syngeneic orthotopic tumour model in reducing tumour burden and silencing PLK1. siPLK1-StAv-SPIONs delivered to PDAC in two clinical relevant mouse models achieve sufficient concentration to be biologically active.

The combination of the favourable accumulation of siPLK1-StAv-SPIONs to tumours and their imaging properties represents an exciting possibility for the simultaneous delivery and detection of siRNA-based therapeutic agents on tumour response in vivo. Drug delivery via siPLK1-StAv-SPIONs can therefore overcome systemic dose limitations of small-molecule inhibitors and might be a promising add-on treatment to conventional therapies such as FOLFIRIBOX in patients with pancreatic cancer.

The feasibility of such studies is underscored by the recent demonstration of substantial silencing in non-human primates following systemic siRNA administration at clinically relevant doses. $^{21}$ One of the main advantages offered by siPLK1-StAv-SPIONs is the possibility to carry out validation studies of selective targets using specific siRNAs at different stages of tumour development. Furthermore, streptavidin/biotin coupling of our ligands gives a higher degree of flexibility in choosing ligands and increases the efficacy of binding (eg, green fluorescent protein (GFP); see online supplementary figure S28). Therefore, we provide a platform that can be modelled according to the tumour specificity and therapeutic target.

Considering that StAv are notorious for being immunogenic, for an application involving human subjects the immunogenicity of StAv must be fully evaluated and may have to be counteracted by use of immunosuppressive drugs, for example, corticosteroids. A frequently raised argument against the use of iron oxide core nanoparticles is their accumulation in the body under repetitive administration. However, there are clinically available intravenous therapeutics such as dextran-coated SPIONs that are already used to treat anaemia without adverse effects, convenient to administer and efficacious at large doses, ${ }^{50}$ which disproves this argument. Moreover, dextran-coated SPIONs, Feridex, are clinically approved for MRI, supporting our conclusion that our probes, which have dextran-coated SPIONs as a backbone, are very well tolerated and biocompatible over longer periods of time.

In conclusion, we believe that our study represents an important new step towards the application of siRNAs as cancer therapeutic agents by providing a new imaging strategy with which to assess their efficacy in tumour-specific silencing and monitoring tumour response.

\section{Author affiliations}

${ }^{1}$ Department of Medicine A, University Medicine, Ernst-Moritz-Arndt-University, Greifswald, Germany

${ }^{2}$ ZIK HIKE-Center for Innovation Competence Humoral Immune Reactions in Cardiovascular Diseases, Greifswald, Germany

${ }^{3}$ Department of Radiology and Neuroradiology, University Medicine, Ernst-MoritzArndt-University Greifswald, Greifswald, Germany

${ }^{4}$ Institute of Pathology, University Medicine, Ernst-Moritz-Arndt-University, Greifswald, Germany

${ }^{5}$ Institute of Pharmacy, Ernst-Moritz-Arndt-University, Greifswald, Germany 
Twitter Follow Raghavendra Palankar at @palankarr

Acknowledgements The authors thank especially David A Tuveson, Kevin Wright, Brianna Creighton and Young-Kyu Park for providing the KPC animals and performing the treatment experiments. Without their help, they would not have been able to perform experiments in the endogenous tumour model.

Contributors MML and JM shared senior authorship. JM, UMM, ST, MS, RP, CvdB and TS were involved in the acquisition of the data, analysis and interpretation of the data and drafting of the manuscript. J-PK served as expert radiologist for the MRI studies. SR and ME helped with TEM and histopathology. GG provided technical expertise in the preparation of nanoparticles. WW, NH, FD, MD and F-UW provided relevant intellectual input. UMM, MML and JM were responsible for study concept and design, drafting of the manuscript, obtained funding and study supervision

Funding This work was supported by the Deutsche Krebshilfe/Dr. Mildred-Scheel-Stiftung (109102), the Deutsche Forschungsgemeinschaft (DFG MA 4115/1-2/3, EV 168/2-1), the Federal Ministry of Education and Research (BMBF GANI-MED 03IS2061A and BMBF 0314107, 01ZZ9603, 01ZZ0103, 01ZZ0403, 03ZIK012) and the European Union (EU-FP-7: EPC-TM and EU-FP7-REGPOT-2010-1).

Competing interests None declared.

Provenance and peer review Not commissioned; externally peer reviewed.

Open Access This is an Open Access article distributed in accordance with the Creative Commons Attribution Non Commercial (CC BY-NC 4.0) license, which permits others to distribute, remix, adapt, build upon this work non-commercially, and license their derivative works on different terms, provided the original work is properly cited and the use is non-commercial. See: http://creativecommons.org/ licenses/by-nc/4.0/

\section{REFERENCES}

1 Maitra A, Leach SD. Disputed paternity: the uncertain ancestry of pancreatic ductal neoplasia. Cancer Cell 2012;22:701-3.

2 Zhang Y, Liu Y, Yang YX, et al. The expression of PLK-1 in cervical carcinoma: a possible target for enhancing chemosensitivity. J Exp Clin Cancer Res 2009:28:130.

3 Yamaguchi T, Goto H, Yokoyama T, et al. Phosphorylation by Cdk1 induces Plk1-mediated vimentin phosphorylation during mitosis. I Cell Biol 2005;171:431.

4 Rizki A, Mott JD, Bissell MJ. Polo-like kinase 1 is involved in invasion through extracellular matrix. Cancer Res 2007;67:11106-10.

5 Gray PJ, Bearss DJ, Han $\mathrm{H}$, et al. Identification of human polo-like kinase 1 as a potential therapeutic target in pancreatic cancer. Mol Cancer Ther 2004;3:641-6.

6 Song B, Liu XS, Rice SJ, et al. Plk1 phosphorylation of orc2 and hbo1 contributes to gemcitabine resistance in pancreatic cancer. Mol Cancer Ther 2013;12:58-68.

7 Raab M, Kappel S, Krämer A, et al. Toxicity modelling of Plk1-targeted therapies in genetically engineered mice and cultured primary mammalian cells. Nat Commun 2011;2:395.

8 Dassie JP, Liu XY, Thomas GS, et al. Systemic administration of optimized aptamer-siRNA chimeras promotes regression of PSMA-expressing tumors. Nat Biotechnol 2009:27:839-46.

9 McNamara JO, Andrechek ER, Wang Y, et al. Cell type specific delivery of siRNAs with aptamer-siRNA chimeras. Nat Biotechnol 2006:24:1005-15.

10 Spankuch B, Matthess $Y$, Knecht $R$, et al. Cancer inhibition in nude mice after systemic application of U6 promoter driven short hairpin RNAs against PLK1. J Natl Cancer Inst 2004;96:862-72.

11 Thorek DL, Chen AK, Czupryna J, et al. Superparamagnetic iron oxide nanoparticle probes for molecular imaging. Ann Biomed Eng 2006;34:23-38.

12 Palmacci S, Josephson L. Synthesis of polysaccharide covered superparamagnetic oxide colloids. USA: Google Patents, 1993.

13 Partecke IL, Kaeding A, Sendler M, et al. In vivo imaging of pancreatic tumours and liver metastases using 7 Tesla MRI in a murine orthotopic pancreatic cancer model and a liver metastases model. BMC Cancer 2011;11:40.

14 Hingorani SR, Wang L, Multani AS, et al. Trp53R172H and KrasG12D cooperate to promote chromosomal instability and widely metastatic pancreatic ductal adenocarcinoma in mice. Cancer Cell 2005;7:469-83.

15 Neesse A, Frese KK, Chan DS, et al. SPARC independent drug delivery and antitumour effects of nab-paclitaxel in genetically engineered mice. Gut 2014:63:974-83.

16 Richardson $\mathrm{KC}$, Jarett L, Finke EH. Embedding in epoxy resins for ultrathin sectioning in electron microscopy. Stain Technol 1960:35:313-23.

17 Sendler M, Dummer A, Weiss FU, et al. Tumour necrosis factor alpha secretion induces protease activation and acinar cell necrosis in acute experimental pancreatitis in mice. Gut 2013;62:430-9.

18 Moore A, Medarova Z, Potthast A, et al. In vivo targeting of underglycosylated MUC-1 tumor antigen using a multimodal imaging probe. Cancer Res 2004;64:1821-7.

19 Hinoda Y, Ikematsu Y, Horinochi M, et al. Increased expression of MUC1 in advanced pancreatic cancer. J Gastroenterol 2003;38:1162-6.
20 Mukherjee P, Ginardi AR, Madsen CS, et al. MUC1-specific CTLs are non-functional within a pancreatic tumor microenvironment. Glycoconj J 2001;18:931-42.

21 Medarova Z, Pham W, Farrar C, et al. In vivo imaging of siRNA delivery and silencing in tumors. Nat Med 2007:13:372-7.

22 Schmit TL, Zhong W, Setaluri V, et al. Targeted depletion of Polo-like kinase (Plk) 1 through lentiviral shRNA or a small-molecule inhibitor causes mitotic catastrophe and induction of apoptosis in human melanoma cells. $J$ Invest Dermatol 2009:129:2843-53.

23 Spankuch-Schmitt B, Bereiter-Hahn J, Kaufmann $M$, et al. Effect of RNA silencing of polo-like kinase-1 (PLK1) on apoptosis and spindle formation in human cancer cells. J Natl Cancer Inst 2002:94:1863-77.

24 Lin CC, Su WC, Yen CJ, et al. A phase I study of two dosing schedules of volasertib (BI 6727), an intravenous polo-like kinase inhibitor, in patients with advanced solid malignancies. Br J Cancer 2014;110:2434-40.

25 Gupta AK, Gupta M. Cytotoxicity suppression and cellular uptake enhancement of surface modified magnetic nanoparticles. Biomateria/s 2005;26:1565-73.

26 Grant BD, Donaldson JG. Pathways and mechanisms of endocytic recycling. Nat Rev Mol Cell Biol 2009; 10:597-608.

27 Conner SD, Schmid SL. Regulated portals of entry into the cell. Nature 2003:422:37-44

28 Kirchhausen T, Macia E, Pelish HE. Use of dynasore, the small molecule inhibitor of dynamin, in the regulation of endocytosis. Meth Enzymol 2008;438:77-93.

29 Dominska M, Dykxhoorn DM. Breaking down the barriers: siRNA delivery and endosome escape. J Cell Sci 2010;123:1183-9.

30 Wang J, Lu Z, Wientjes MG, et al. Delivery of siRNA therapeutics: barriers and carriers. AAPS J 2010;12:492-503.

31 Chiu YL, Ali A, Chu CY, et al. Visualizing a correlation between siRNA localization, cellular uptake, and RNAi in living cells. Chem Biol 2004:11:1165-75.

32 Bulte JW, Zhang S, van Gelderen P, et al. Neurotransplantation of magnetically labeled oligodendrocyte progenitors: magnetic resonance tracking of cell migration and myelination. Proc Natl Acad Sci USA 1999;96:15256-61.

33 Noetzli LJ, Papudesi J, Coates TD, et al. Pancreatic iron loading predicts cardiac iron loading in thalassemia major. Blood 2009;114:4021-6.

34 Gupta AK, Gupta M. Synthesis and surface engineering of iron oxide nanoparticles for biomedical applications. Biomaterials 2005;26:3995-4021.

35 Zhang Y, Chen L, Yang J, et al. Study human pancreatic cancer in mice: how close are they? Biochim Biophys Acta 2013;1835:110-18.

36 Copolovici DM, Langel K, Eriste E, et al. Cell-penetrating peptides: design, synthesis, and applications. ACS Nano 2014;8:1972-94.

37 Raynal I, Prigent P, Peyramaure $S$, et al. Macrophage endocytosis of superparamagnetic iron oxide nanoparticles: mechanisms and comparison of ferumoxides and ferumoxtran-10. Invest Radiol 2004;39:56-63.

38 Yezhelyev MV, Qi LF, O'Regan RM, et al. Proton-sponge coated quantum dots for siRNA delivery and intracellular imaging. J Am Chem Soc 2008;130: 9006-12.

39 Veiseh O, Kievit FM, Mok H, et al. Cell transcytosing poly-arginine coated magnetic nanovector for safe and effective siRNA delivery. Biomaterials 2011:32:5717-25.

40 Varkouhi AK, Scholte M, Storm G, et al. Endosomal escape pathways for delivery of biologicals. J Control Release 2011;151:220-8.

41 Jimeno A, Li J, Messersmith WA, et al. Phase I study of ON 01910. Na, a novel modulator of the Polo-like kinase 1 pathway, in adult patients with solid tumors. $J$ Clin Oncol 2008;26:5504-10.

42 Mross K, Dittrich C, Aulitzky WE, et al. A randomised phase II trial of the Polo-like kinase inhibitor BI 2536 in chemo-naïve patients with unresectable exocrine adenocarcinoma of the pancreas a study within the Central European Society Anticancer Drug Research (CESAR) collaborative network. Br I Cancer 2012;107:280-6.

43 Gjertsen BT, Schöffski P. Discovery and development of the Polo-like kinase inhibitor volasertib in cancer therapy. Leukemia 2015;29:11-19.

44 Bartlett DW, Davis ME. Insights into the kinetics of siRNA-mediated gene silencing from live-cell and live-animal bioluminescent imaging. Nucleic Acids Res 2006:34:322-33

45 Medarova Z, Pham W, Kim Y, et al. In vivo imaging of tumor response to therapy using a dual-modality imaging strategy. Int I Cancer 2006;118:2796-802.

46 Bibby MC. Orthotopic models of cancer for preclinical drug evaluation: advantages and disadvantages. Eur I Cancer 2004;40:852-7.

47 Frese KK, Tuveson DA. Maximizing mouse cancer models. Nat Rev Cancer 2007;7:645-58

48 Olive KP, Jacobetz MA, Davidson CJ, et al. Inhibition of Hedgehog signaling enhances delivery of chemotherapy in a mouse model of pancreatic cancer. Science 2009;324:1457-61.

49 Van Dyke T. Finding the tumor copycat: approximating a human cancer. Nat Med 2010;16:976-7.

50 Auerbach $\mathrm{M}$, Winchester J, Wahab $\mathrm{A}$, et al. A randomized trial of three iron dextran infusion methods for anemia in EPO-treated dialysis patients. Am J Kidney Dis 1998;31:81-6. 